\title{
Dipolar magnets and glasses: Neutron-scattering, dynamical, and calorimetric studies of randomly distributed Ising spins
}

\author{
D. H. Reich, ${ }^{*}$ B. Ellman, J. Yang, and T. F. Rosenbaum \\ The James Franck Institute and Department of Physics, The University of Chicago, Chicago, Illinois 60637 \\ G. Aeppli \\ AT\&T Bell Laboratories, Murray Hill, New Jersey 07974 \\ D. P. Belanger ${ }^{\dagger}$ \\ Physics Department, Brookhaven National Laboratory, Upton, New York 11973
}

(Received 23 April 1990)

\begin{abstract}
We have measured the magnetic correlations, susceptibility, specific heat, and thermal relaxation in the dipolar-coupled Ising system $\mathrm{LiHo}_{x} \mathrm{Y}_{1-x} \mathrm{~F}_{4}$. The material is ferromagnetic for spin concentrations at least as low as $x=0.46$, with a Curie temperature obeying mean-field scaling relative to that of pure $\mathrm{LiHoF}_{4}$. In contrast, an $x=0.167$ sample behaves as a spin glass above its transition temperature, while an $x=0.045$ crystal shows very different glassy properties characterized by decreasing barriers to relaxation and nonexponential thermal relaxation as $T \rightarrow 0$. We find the properties of the $x=0.045$ system to be consistent with a single low-degeneracy ground state with a large gap for excitations. The $x=0.167$ sample, however, supports a complex ground state with no appreciable gap, in accordance with prevailing theories of spin glasses. The underlying causes of such disparate behavior are discussed in terms of random clusters as probed by neutron studies of the $x=0.167$ sample.
\end{abstract}

\section{INTRODUCTION}

The diverse variety of glassy systems, the historydependent nature of their response, and the multiplicity of allowable ground states make it difficult to identify the salient microscopic properties of glassy behavior. One approach to this problem is to find a simple prototype that has many or all of the features of "canonical" glasses and yet is easy to characterize microscopically. With that intent, we report frequency-dependent magnetic susceptibility, specific-heat, thermal relaxation, and neutron-scattering measurements, of the dilute, insulating, dipolar-coupled Ising magnet ${ }^{1-4} \mathrm{LiHo}_{x} \mathrm{Y}_{1-x} \mathrm{~F}_{4}$. Here, Ising dipoles occupy randomly chosen sites of a regular lattice. The form of the microscopic Hamiltonian is completely understood, but the anisotropy and longrange nature of the dipolar interaction, combined with the disorder introduced by replacing the magnetic $\mathrm{Ho}^{3+}$ ions with nonmagnetic $\mathrm{Y}^{3+}$, provide for a rich spectrum of physical responses. In particular, we show that different regimes of magnetic glassiness can be accessed by varying the holmium concentration $x$.

For Ising spins, the dipole-dipole interaction is given by

$$
E_{i j}=s_{i} s_{j} J\left(1-3 \cos ^{2} \theta_{i j}\right) / r_{i j}^{3},
$$

where $s_{i}$ and $s_{j}= \pm 1$ are the Ising spin variables, $J=g^{2} \mu_{B}^{2}$ is the coupling constant, $\theta_{i j}$ is the angle formed by the Ising axis and the vector connecting the spins, and $r_{i j}$ is the interspin distance. The radial dependence, $E_{i j} \propto 1 / r_{i j}^{3}$, means that many more than nearest-neighbor interactions must be taken into account. The angular dependence, $E_{i j} \propto\left(1-3 \cos ^{2} \theta_{i j}\right)$, means that $E_{i j}$ can change sign. Specifically, for angles close to the Ising axis, the interaction is ferromagnetic, but for intermediate values $\left(55^{\circ} \leq \theta_{i j} \leq 125^{\circ}\right)$ it is antiferromagnetic. Early theoretical work $\mathrm{k}^{5-8}$ on this problem focused on whether, in the face of these competing interactions, dipolar ferromagnetism can occur at all, and a classical analysis by Luttinger and Tisza $^{7}$ showed that even at zero temperature the ordering depends both on lattice structure and sample shape.

With the development of the renormalization-group (RG) theory, there was a resurgence of interest in dipolar magnets. It was shown ${ }^{9,10}$ that for a dipolar-coupled Ising system the marginal dimensionality $d^{*}$ that separates classical, mean-field-type behavior from nonclassical behavior is three. For most other systems, $d^{*} \geq 4$. Thus, the dipolar, Ising magnet represents one of the few cases where predictions based on exact solutions of the RG equations at $d=d^{*}$ can be tested experimentally.

It was soon noted ${ }^{2,11}$ that in the compounds $\mathrm{Li} R \mathrm{~F}_{4}$, where $R$ is a rare-earth element, the rare-earth elements are mostly dipolar coupled, and that the ferromagnets $\mathrm{LiTbF}_{4}\left(\right.$ Curie temperature $\left.T_{C}=2.86 \mathrm{~K}\right)$ and $\mathrm{LiHoF}_{4}$ $\left(T_{C}=1.53 \mathrm{~K}\right)$ are essentially Ising at low temperatures. This led to an extensive series of investigations by various groups, ${ }^{12-18}$ first on $\mathrm{LiTbF}_{4}$ and then on $\mathrm{LiHoF}_{4}$, which found the predicted logarithmic corrections $\mathrm{s}^{9,19}$ to meanfield exponents near $T_{C}$ in the susceptibility and specific heat.

An important feature of the rare-earth lithium fluorides is that a nonmagnetic impurity, yttrium, can be 
introduced substitutionally, producing a dilution series $\mathrm{Li}_{x} \mathrm{Y}_{1-x} \mathrm{~F}_{4}$. This raises the possibility of studying a random, dipolar-coupled Ising system. We are aware of only two theoretical works on this question. In one, Aharony ${ }^{20}$ did an $R G$ analysis of the critical behavior of the random, dipolar-coupled Ising model that predicted corrections to mean-field exponents at $d^{*}=3$ different from those for the undiluted case. In the other, Aharony and Stephen ${ }^{21}$ studied the variation of the ordering temperature $T_{C}$ with spin concentration $x$ for various longrange interactions in the $x \rightarrow 0$ limit of the random Ising model. Their work contains the suggestion that, for dipolar interactions, spin-glass ordering may be favored over ferromagnetic ordering for $x$ less than some critical concentration $x_{c}$.

Inspired by the first of these papers, various investigators $^{22,23}$ studied the $\mathrm{LiTb}_{x} \mathrm{Y}_{1-x} \mathrm{~F}_{4}$ series. The ground state of a $\mathrm{Tb}^{3+}$ ion in the crystal field of the $\mathrm{LiRF}_{4}$ lattice is not a true Ising doublet, but rather a pair of singlets ${ }^{2}$ separated by $1.2 \mathrm{~K}$. Thus, $\mathrm{LiTbF}_{4}$ is an induced moment ferromagnet, ${ }^{24}$ and the properties of the dilution series are manifestations of a crossover to Van Vleck paramagnetism rather than a direct consequence of randomness. Since the ground state of the $\mathrm{Ho}^{3+}$ in the $\mathrm{Li}_{x} \mathrm{Y}_{1-x} \mathrm{~F}_{4}$ lattice is a true Ising doublet, $\mathrm{LiHo}_{x} \mathrm{Y}_{1-x} \mathrm{~F}_{4}$ is a better system to study in the context of randomly distributed dipoles.

In this paper, we describe magnetic susceptibility, specific-heat, thermal relaxation, and neutron-scattering experiments on $\mathrm{LiHo}_{1-x} \mathrm{Y}_{1-x} \mathrm{~F}_{4}$ with $x=0.67,0.46$, 0.167 , and 0.045 . As a function of decreasing dipole concentration, the system is characterized by qualitatively different ground states: ferromagnetic (FM), spin glass (SG), and "decoupled cluster glass" (DCG). This latter state, in the dilute limit, does not appear to freeze at finite temperature, and in view of the long-range nature of the

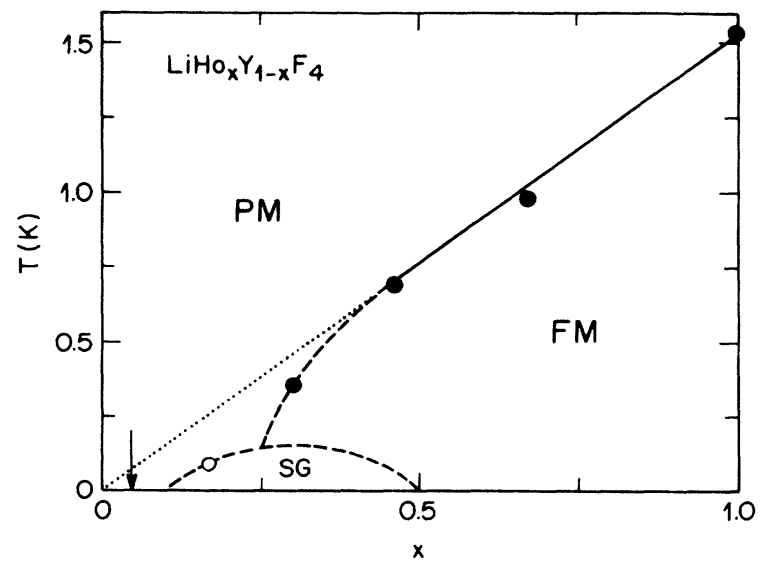

FIG. 1. Phase diagram for the diluted dipolar-coupled Ising magnet, $\mathrm{LiHo}_{x} \mathrm{Y}_{1-x} \mathrm{~F}_{4}$. $\mathrm{PM}=$ Paramagnet, $\mathrm{FM}=$ Ferromagnet, $\mathrm{SG}=$ Spin Glass. Arrow shows location of "decoupled cluster glass," described in text. Solid circles are from neutron and magnetic susceptibility measurements (this work, Ref. 3, and Ref. 51). The open circle denotes a spin-glass transition temperature obtained from a dynamic scaling analysis of the $x=0.167$ susceptibility data. dipolar interaction, is least expected. We show in Fig. 1 the magnetic phase diagram for $\mathrm{LiHo}_{x} \mathrm{Y}_{1-x} \mathrm{~F}_{4}$ inferred from the current experiments as well as experience with other systems exhibiting both FM and SG states. ${ }^{25,26}$

The paper is organized as follows. In the next section we describe sample properties and measurement techniques. Section III presents the experimental results and data analysis, while Sec. IV contains a discussion which attempts to relate the different measurements to particular ground-state and excited-state structures. Finally, Sec. V summarizes the discoveries made in the course of our study.

\section{EXPERIMENTAL METHODS}

\section{A. Properties of $\mathrm{LiHo}_{x} \mathrm{Y}_{1-x} \mathrm{~F}_{4}$}

The ${\mathrm{Li} R \mathrm{~F}_{4}}_{4}$ compounds have a body-centered, tetragonal unit cell containing four formula units, ${ }^{2}$ shown in Fig. 2. The lattice constants ${ }^{1}$ are $a=a^{\prime}=5.175(5) \AA$ and $c=10.75$ (1) $\AA$. The magnetic holmium ions compete for the same lattice sites as the nonmagnetic yttrium ions. Thus, by varying the [Ho]:[Y] ratio, differing amounts of randomness can be introduced into the Ho spin system.

The $\mathrm{Ho}^{3+}$ ions in this system constitute a nearly ideal dipolar-coupled Ising system at low temperatures. The ground state of a $\mathrm{Ho}^{3+}$ ion in the crystal field of the $\mathrm{Li} R \mathrm{~F}_{4}$ lattice is an Ising doublet, ${ }^{3}$ but with $g=14$. The Ising axis is the $c$ axis of the crystal. The first excited crystal-field level is $9.4 \mathrm{~K}$ above the ground-state doublet; at the low temperatures of our experiments, $T \leq 1 \mathrm{~K}$, only the Ising doublet is appreciably populated. These materials are optically transparent insulators, so there is no Ruderman-Kittel (-Kasuya)-Yosida (RKKY) interaction between the Ho ions. In addition, exchange couplings between the $\mathrm{Ho}^{3+}$ ions are small; the exchange energy for nearest neighbors is half that of the nearest-

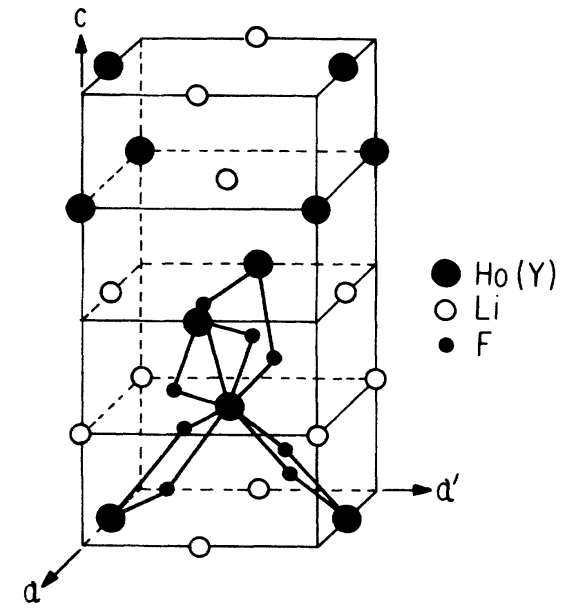

FIG. 2. Unit cell of $\mathrm{LiHo}_{x} \mathrm{Y}_{1-x} \mathrm{~F}_{4}$, space group $C^{6}{ }^{4 h}\left(I 4_{1} / a\right)$. Lattice constants are $a=a^{\prime}=5.175(5) \AA$ and $c=10.75(1) \AA$. Ho and $\mathrm{Y}$ atoms compete for the lattice sites designated by large, solid circles. Fluorine atoms are only shown around the $\mathrm{Ho}(\mathrm{Y})$ site at $(a / 2, a / 2, c / 4)$ (following Ref. 2). 
neighbor dipolar interaction, and for second nearest neighbors it is only $5 \%$ of the dipolar interaction. ${ }^{2}$ Thus, the magnetic properties of $\mathrm{LiHo}_{x} \mathrm{Y}_{1-x} \mathrm{~F}_{4}$ are dominated by the dipolar forces, as confirmed by our neutronscattering measurements (see below). The nearestneighbor dipolar interaction is ferromagnetic; the second-nearest-neighbor interaction is antiferromagnetic. Pure $\mathrm{LiHoF}_{4}$ is a ferromagnet ${ }^{2}$ with $T_{C}=1.53 \mathrm{~K}$, but there is a delicate balance between competing interactions in this crystal structure, and one expects the effects of dilution to be pronounced.

$\mathrm{LiHo}_{x} \mathrm{Y}_{1-x} \mathrm{~F}_{4}$ is also an attractive material to work with for a number of purely practical reasons. Since it is an insulator, ac susceptibility measurements can be extended to very high frequencies without having to worry about eddy-current heating, as in a metal. $\mathrm{LiHo}_{x} \mathrm{Y}_{1-x} \mathrm{~F}_{4}$ is used for lasing purposes, so large, single crystals are commercially available. Our samples, single crystals of order $0.25 \mathrm{~cm}^{3}$ in size and $1 \mathrm{~g}$ in mass, were either purchased from Sanders Associates (Nashua, New Hampshire), or obtained from Howard Guggenheim (AT\&T Bell Labs). Chemical segregation effects have been explicitly ruled out in these samples by neutronscattering studies. For example, the upper bound for the full width at half maximum (FWHM) of the $(1.98,0,0)$ peak in an $x=0.67$ ferromagnetic sample with $T_{c}=0.98$ $\mathrm{K}$ is $10 \mathrm{mK}$ (see below), even disregarding finite- $q$ broadening and limitations due to instrumental resolution. Hence, concentration fluctuations must be less than one percent. $\mathrm{LiHo}_{x} \mathrm{Y}_{1-x} \mathrm{~F}_{4}$ is optically active, so the $c$ axis can be found merely by viewing a crystal between crossed polarizers. Finally, the lattice constants vary by less than $0.01 \%$ between $x=0$ and $1 .{ }^{1}$ This means that one can determine the Ho concentration $x$ to $\pm 0.1 \%$ by an accurate measurement of a sample's density. We used an air-water differential weighing technique ${ }^{27}$ to that end.

\section{B. Neutron diffraction}

We performed neutron-scattering experiments at the cold neutron facility of the Brookhaven High Flux Beam Reactor. A single crystal of $\mathrm{LiHo}_{0.167} \mathrm{Y}_{0.833} \mathrm{~F}_{4}$, with dimensions $6 \times 6 \times 6 \mathrm{~mm}^{3}$, was placed in a dilution refrigerator which was mounted on a triple-axis neutron spectrometer equipped with pyrolitic graphite double monochromator and analyzer crystals. The collimation was 60', 40', 60', 80', open, in the core, monochromator, monochromator-sample, sample-analyzer, and analyzerdetector positions, respectively. Be filters eliminated higher-order beam contamination. With incident and final neutron energies of $5 \mathrm{meV}$, we measured the diffuse, magnetic scattering near the $(2,0,0)$ Bragg peak of $\mathrm{LiHo}_{0.167} \mathrm{Y}_{0.833} \mathrm{~F}_{4}$ for temperatures between 0.15 and 1.5 $\mathbf{K}$, as given by a carbon thermometer mounted next to the sample. The neutron scan intensity was normalized to 5000 counts detected at the monitor, representing a counting time of approximately five minutes. A background signal of six counts per minute was subtracted from the data. We also measured the temperature dependence of the scattering from a ferromagnetic sample with $x=0.67$. The sample, an irregularly shaped single crys- tal with an approximate volume of $0.5 \mathrm{~cm}^{3}$, was cooled in a ${ }^{3} \mathrm{He}$ cryostat. The collimation was $30^{\prime}, 40$ ', 30', 40', 40', and the neutron energy was $4.5 \mathrm{meV}$. Temperature scans at $(2,0,0)$ and $(1.98,0,0)$ were taken to measure the magnetic Bragg scattering and the critical scattering near $T_{C}$, respectively.

\section{Magnetic susceptibility}

We used a variety of phase-sensitive inductance bridge techniques to measure the real and imaginary parts of the ac magnetic susceptibility,

$$
\chi(\omega, T)=\chi^{\prime}(\omega, T)+i \chi^{\prime \prime}(\omega, T),
$$

at millikelvin temperatures. Initially, we mapped out the phase diagram of $\mathrm{LiHo}_{x} \mathrm{Y}_{1-x} \mathrm{~F}_{4}$ by measuring $\chi(T)$ at fixed $\omega$ for samples of differing Ho concentration. In order to change samples quickly, we used the top-loading capability of the dilution refrigerator. Thus, the coils were constrained to fit in the $0.855 \mathrm{in}$. long $\times 0.325 \mathrm{in}$. diameter, semicylindrical cutout of the top-loading sample holder, and fit snugly around the samples, each of order $0.5 \times 0.5 \times 1.0 \mathrm{~cm}^{3}$ in size. Typical coils were made of 225 turns of 40-gauge $\mathrm{Cu}$ wire for the secondary and 150 turns of 36-gauge Cu wire for the primary. With the primary approximately three times as long as the secondary, the magnetic field $H \leq 3.2 \times 10^{-3}$ Oe was homogeneous to $\approx 3 \%$ over the length of the sample. An external bridge circuit ${ }^{28}$ was used to balance the voltage induced in the secondary, which was transformer coupled to a PAR 124A lockin amplifier.

More sensitive measurements were required to investigate the glassy dynamics of the two most dilute samples, $x=0.045$ and 0.167 . Spectroscopic studies were made of a $0.95 \times 0.52 \times 0.52 \mathrm{~cm}^{3}$ single crystal of $\mathrm{LiHo}_{0.045} \mathrm{Y}_{0.955} \mathrm{~F}_{4}$ with the Ising axis parallel to its length. Here, the bridge contained two identical mutual inductances bolted to the mixing chamber of the dilution refrigerator, with the secondary coils wired in opposition. The primaries were $5 \mathrm{~cm}$ long, containing 1460 turns of 36-gauge $\mathrm{Cu}$ wire in four layers, and provided a field $H<0.04$ Oe that was uniform to $1 \%$ over the length of the sample. The secondaries each had 2750 turns of 40 gauge $\mathrm{Cu}$ wire wound in 25 layers around a Mylar form of the same dimensions as the sample. We measured the magnetic response, $\chi(\omega)$, at eight temperatures between 90 and $300 \mathrm{mK}$. Data were obtained from $0.2 \mathrm{~Hz}$ to 20 $\mathrm{kHz}$ with a PAR 124A lockin amplifier, and from 0.02 to $2 \mathrm{~Hz}$ with a combination HP 3325A frequency synthesizer, computer-based digital lockin technique. ${ }^{29}$ Similarly, using a combination of conventional and computer-based digital lockin amplifiers, we measured the complex ac susceptibility of a single crystal of $\mathrm{LiHo}_{0.167} \mathrm{Y}_{0.833} \mathrm{~F}_{4}$ at fixed temperature between 0.12 and $0.3 \mathrm{~K}$ over the frequency range $0.5 \mathrm{~Hz}$ to $10 \mathrm{kHz}$. The sample was cut to a $5.0 \times 0.75 \times 0.75 \mathrm{~mm}^{3}$ needle in order to reduce demagnetization effects, with its Ising (c) axis along its length. Data was collected with a SQUID susceptometer and inductance bridge. The frequency range was limited by noise at the low end and by instabilities in the SQUID electronics at the high end. The arm of the bridge con- 
taining the sample consisted of a primary (excitation) coil of 310 turns of superconducting $\mathrm{NbTi}$ wire with two secondary (pickup) coils each of 7 turns of NbTi wire, also arranged in a gradiometer configuration. The coils were placed in a lead shield and mounted on the mixing chamber of the dilution refrigerator, with the sample heat sunk to the mixing chamber via 36-gauge $\mathrm{Cu}$ wire silverepoxied to its ends. The field from the primary was $2.0 \times 10^{-4}$ Oe and was calculated to vary by $<0.5 \%$ over the length of the sample. The magnetic response for all samples was always in the linear regime, in contrast to recent studies of electric dipolar glasses where the polarization was found to be a nonanalytic function of field. ${ }^{30}$

\section{Calorimetry}

Specific-heat measurements using both pulse and relaxation techniques were performed on approximately 30$\mathrm{mg}$ single-crystal platelets of both $\mathrm{LiHo}_{0.045} \mathrm{Y}_{0.955} \mathrm{~F}_{4}$ and $\mathrm{LiHo}_{0.167} \mathrm{Y}_{0.833} \mathrm{~F}_{4}$. The platelet was greased with Apiezon $\mathrm{N}$ to a single-crystal sapphire substrate with a gold heater evaporated on its back. The thermometer was a chip cut from a $220 \Omega$ carbon Spear thermometer calibrated against a germanium thermometer on the mixing chamber. We estimate that the addendum was less than $1 \%$ of the total signal at $1 \mathrm{~K}$ and negligible at the lowest $T$. Heat pulses yielded temperature excursions never in excess of $5 \mathrm{mK}$ at $0.13 \mathrm{~K}$ and $20 \mathrm{mK}$ at $1.5 \mathrm{~K}$. Care was taken to allow the samples to reach equilibrium over many hours at the lowest temperatures before initiating the measurements.

\section{RESULTS}

\section{A. Overview}

Neutron-scattering and ac susceptibility measurements as a function of temperature reveal a crossover from ferromagnetism to glassiness with increasing dilution of the dipoles. Magnetic Bragg scattering from $\mathrm{LiHo}_{0.67} \mathrm{Y}_{0.33} \mathrm{~F}_{4}$, plotted in Fig. 3, clearly indicates ferromagnetic ordering. The growth of the order parameter for $T<T_{C}$ is shown in Fig. 3(b), while the temperature scan in Fig. 3(a), taken for a wave vector slightly displaced from the ordering vector, shows the divergence of the susceptibility at $T_{c}=0.98 \mathrm{~K}$. The background rising below $T_{c}$ in Fig. 3(a) arises from domain-wall scattering, which is generally pronounced in Ising ferromagnets.

Evidence for magnetic ordering can also be obtained from the bulk ac susceptibility. We plot in Fig. $4 \chi^{\prime}(T)$ at $f=50 \mathrm{~Hz}$ for $\mathrm{LiHo}_{0.46} \mathrm{Y}_{0.54} \mathrm{~F}_{4}$. As $T$ decreases, $\chi^{\prime}$ first increases to the demagnetizing $\operatorname{limit}^{31}$ at $T_{C}=0.68 \mathrm{~K}$, then abruptly drops, as expected for Ising ferromagnets. For both of these concentrations, $T_{C}$ satisfies the meanfield prediction $T_{C}(x)=x T_{C}(x=1)$ within the errors in measuring temperature and concentration. We illustrate this point in Fig. 1, where the measured Curie temperatures (solid circles) follow the mean-field law (solid line).

With further dilution, $\mathrm{LiHo}_{x} \mathrm{Y}_{1-x} \mathrm{~F}_{4}$ behaves very

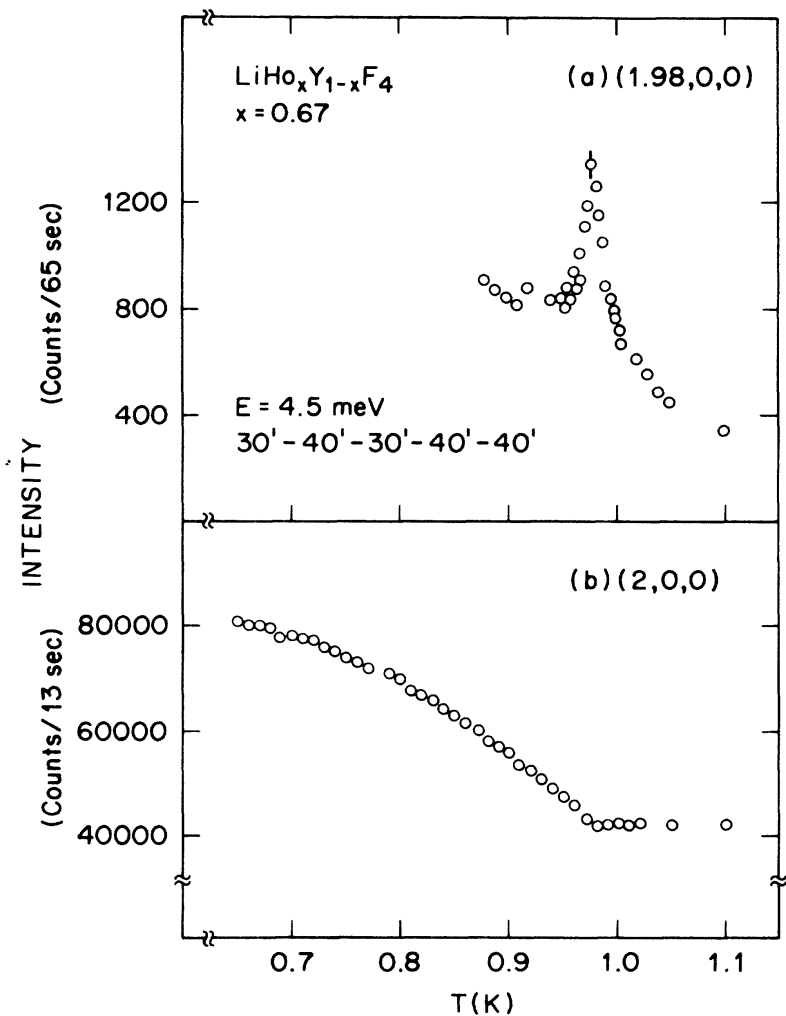

FIG. 3. Neutron-scattering intensity vs temperature for $\mathrm{LiHo}_{0.67} \mathrm{Y}_{0.33} \mathrm{~F}_{4}$, a ferromagnet with $T_{C}=0.98 \mathrm{~K}$. (a) Scattering with wave vector slightly off the $(2,0,0)$ Bragg peak, showing divergence of $\chi$ at $T_{C}$. Vertical line at peak shows size of (statistical) error bar. (b) Magnetic Bragg scattering at $(2,0,0)$ showing onset of spontaneous magnetization at $T_{C}$.

differently than expected for a mean-field dipolar ferromagnet. Figure 5 shows $\chi^{\prime}(T)$ at $f=50 \mathrm{~Hz}$ for $x=0.167$ and $x=0.045$. For these data, as for $x=0.46$ above, the vertical scales were calibrated by fitting $\chi^{\prime}$ to a Curie law at high $T$, using a moment of $7 \mu_{B}$ per $\mathrm{Ho}^{3+}$ ion. The data for $x=0.045$ and 0.167 have several im-

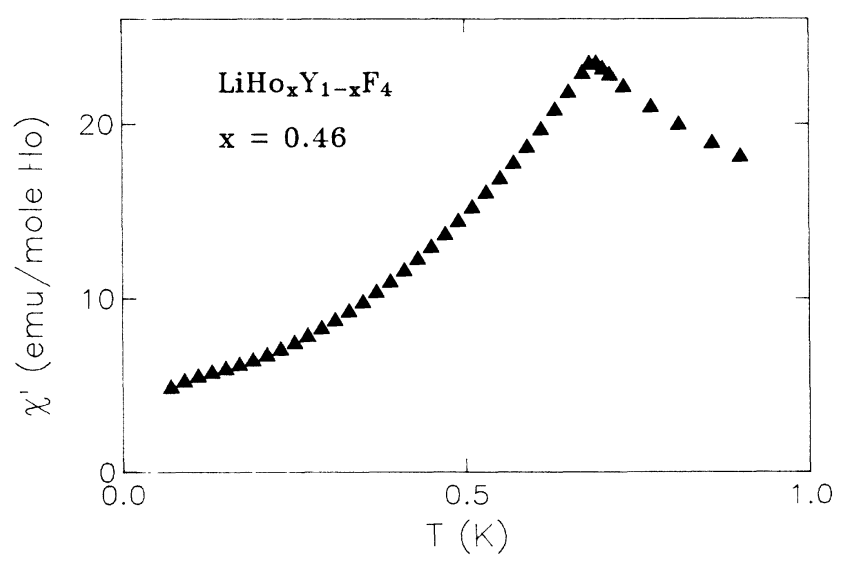

FIG. 4. Real part of the ac magnetic susceptibility, $\chi^{\prime}$, as a function of temperature $T$ at $f=50 \mathrm{~Hz} . \mathrm{LiHo}_{0.46} \mathrm{Y}_{0.54} \mathrm{~F}_{4}$ appears to be a conventional ferromagnet with a (mean-field) reduced $T_{c}$ (following Ref. 4). 


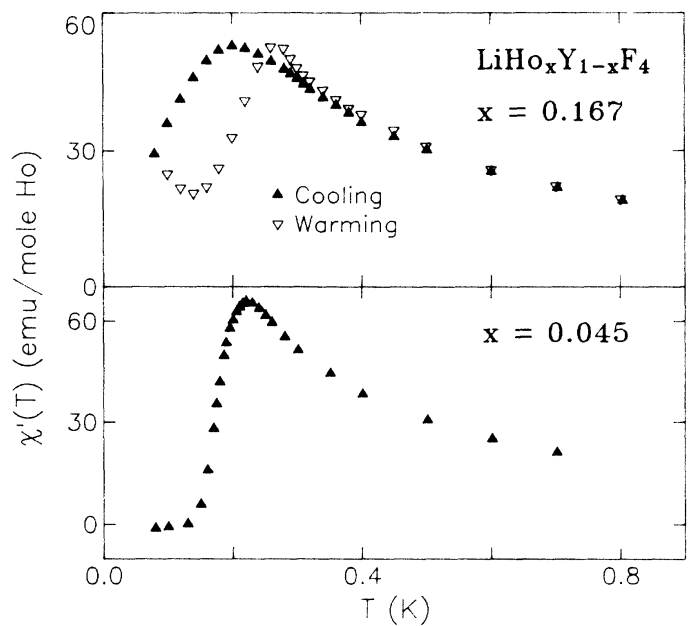

FIG. 5. Real part of the ac magnetic susceptibility, $\chi^{\prime}$, as a function of temperature, $T$, at $f=50 \mathrm{~Hz}$. Both samples appear to be glassy magnets, where the equilibrium time of the more concentrated $\mathrm{LiHo}_{0.167} \mathrm{Y}_{0.833} \mathrm{~F}_{4}$ is of order a day per point (following Ref. 4).

portant features. First, long-time processes become apparent at low $T$. Equilibrium data can be obtained for the $x=0.045$ sample only by cooling or warming in zero applied field at rates less than $20 \mathrm{mK} / 2 \mathrm{~h}$; cooling and warming curves do not coincide for the $x=0.167$ sample even then. Furthermore, the real and imaginary parts of the susceptibility are of comparable magnitude for these two concentrations, ${ }^{4}$ which indicates that the magnetic relaxation occurs at rates of order the measuring frequency $(50 \mathrm{~Hz})$. Second, the maximal value of $\chi^{\prime}(T)$ is at only $\frac{2}{3}$ and $\frac{1}{5}$ the demagnetizing limit for the $x=0.167$ and 0.045 samples, respectively. ${ }^{31}$ Third, $\chi^{\prime}(T)$ peaks at temperatures well above the mean-field expectation, $x T_{C}(x=1)$, suggesting that the long-time glassy behavior preempts the ferromagnetism. Surprisingly, this new temperature scale does not seem to scale with spin concentration between $x=0.045$ and 0.167 . Finally, a pronounced measuring frequency dependence arises, as shown in Fig. 6 for the most dilute sample. With increasing $T$ the $\chi^{\prime}(T)$ curves begin to overlap for progressively higher frequencies. Concomitantly, as $f$ is reduced, the maximum in $\chi^{\prime}(T)$ both moves to lower $T$ and sharpens. The peak frequencies, $f_{p}$, are well described by the Arrhenius law familiar from studies of glasses,

$$
f_{p}=1 / \tau_{\text {char }}=f_{0} \exp \left(-E_{B} / k T\right),
$$

at least over our limited frequency and temperature range (inset). The solid line is a least-squares fit with $f_{0}=1.0 \times 10^{6} \mathrm{~Hz}$ and $E_{B} / k=2.2 \mathrm{~K}$. This energy is of order the nearest-neighbor interaction energy and, as pointed out by Atsarkin, ${ }^{32}$ of order the width of the nuclear hyperfine multiplet. The extraordinarily low attempt frequency, $f_{0}$, for this Ising spin system at low temperatures presumably accounts for the observation of hysteresis in the ac susceptibility. ${ }^{33}$ From Eq. (2), $\tau_{\text {char }}=1 \mathrm{~h}$ for $T=0.1 \mathrm{~K}$.

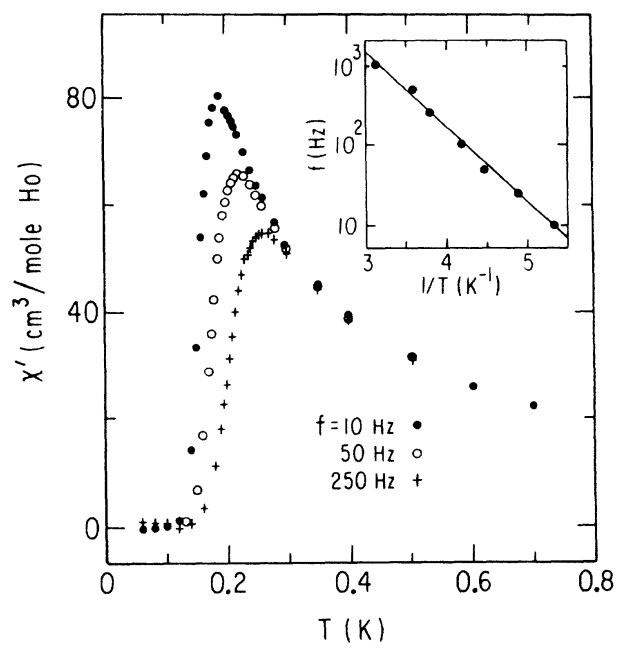

FIG. 6. Frequency, $f$, dependence of $\chi^{\prime}(T)$ for $\mathrm{LiHo}_{0.045} \mathrm{Y}_{0.955} \mathrm{~F}_{4}$. The peak in $\chi^{\prime}$ follows an Arrhenius law (inset), typical of glasses (from Ref. 4).

\section{B. Spectroscopic response}

The Arrhenius parameters are the essential physical information derived from temperature scans of the ac magnetic susceptibility at fixed frequency. In order to discern more than the temperature evolution of the peak frequency, one can map out the spectral response by fixing $T$ and varying $\omega$. Due to the myriad modes of relaxation in a glassy system, this approach requires sampling a wide range of time scales. We plot in Fig. 7 the real and imaginary parts of the susceptibility for $x=0.045$ over six decades in frequency at four different temperatures. In addition to providing a picture of the full dynamics of the system, this approach offers the advantage of more easily accommodating the extraordinarily long thermal relaxation times at temperatures below the peak in $\chi^{\prime}(T)$. After

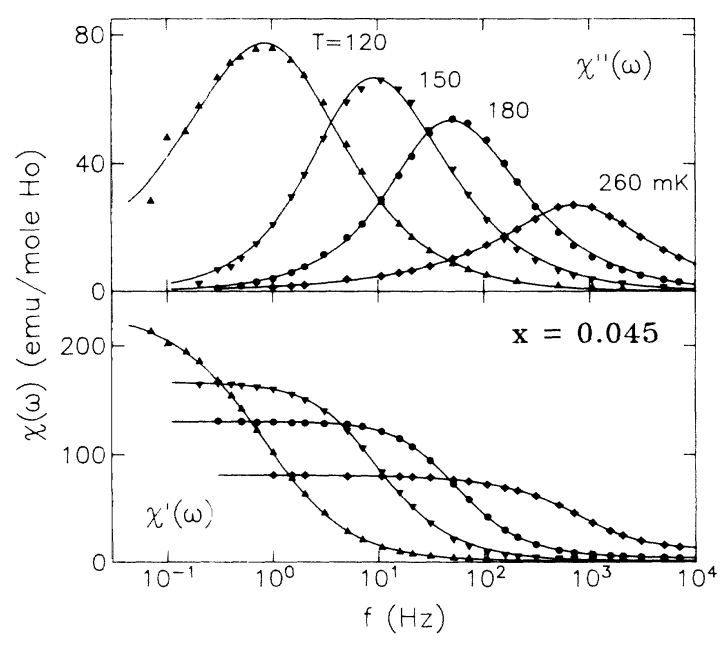

FIG. 7. Real $\left(\chi^{\prime}\right)$ and imaginary $\left(\chi^{\prime \prime}\right)$ parts of the magnetic susceptibility of $\mathrm{LiHo}_{0.045} \mathrm{Y}_{0.955} \mathrm{~F}_{4}$ vs frequency $f$ for several temperatures $T$. Solid lines are fits to a distribution of energy barriers to relaxation described in the text (following Ref. 40). 
equilibrating at one $T$, frequency can be scanned at will. Hence, we were able to obtain equilibrium data for the $x=0.167$ sample, plotted in Fig. 8. At each $T$, the peak in $\chi^{\prime \prime}(\omega)$ coincides roughly with the inflection point in $\chi^{\prime}(\omega)$. This peak follows an Arrhenius law over more than four decades in frequency ${ }^{34}$ (giving slightly different parameters over this larger range than the $\chi^{\prime}(T)$ data for $x=0.045$ ), with $f_{0}=2.0 \times 10^{5} \mathrm{~Hz}$ and $E_{B} / k=1.46 \mathrm{~K}$ for $x=0.045$, and $f_{0}=8.25 \times 10^{6} \mathrm{~Hz}$ and $E_{B} / k=2.05 \mathrm{~K}$ for $x=0.167$. Both data sets are corrected for demagnetization effects by approximating the shape of the samples by a prolate ellipsoid of revolution. The solid lines are nonlinear-least-squares fits described in detail below.

Extrapolating $\chi^{\prime}$ to $\omega=0$ in Figs. 7 and 8 allows a reliable determination of the dc susceptibility for $T \geq 0.15$ K. The small characteristic microscopic frequency, $f_{0}$, of these Ising systems at low $T$ makes it difficult to ascertain the dc limit at the lowest temperatures using this approach. We find that the static magnetic susceptibilities in the two crystals evolve quite similarly, and scale simply with dipole concentration at higher $T$, as can be seen in Fig. 9, which shows $1 / \chi^{\prime}(\omega \rightarrow 0)$. At high $T$, the data for both samples follow Curie-Weiss laws with Curie constants given by the free-spin moments and intercepts $\theta=0.05$ and $0.16 \mathrm{~K}$ for $x=0.045$ and 0.167 , respectively. Both values for $\theta$ are somewhat below the expected (mean-field) intercepts. Furthermore, for $x=0.167$, there are clear deviations from Curie-Weiss behavior at low $T$, confirming that this sample, unlike crystals with larger $x$, is not subject to a ferromagnetic instability. The deviations from the Curie-Weiss law begin at $T \approx 0.25 \mathrm{~K}$. This corresponds to the ferromagnetic transition temperature predicted by a mean-field-type scaling of $T_{c}$ with concentration: $x T_{c}(x=1)=0.167 \times 1.53 \mathrm{~K}=0.255 \mathrm{~K}$, and may be related to the Griffiths point in spin glasses, ${ }^{35}$ the rough temperature below which collective magnetic effects appear. From the positive sign of the Curie-Weiss temperature, $\Theta$, we infer that ferromagnetic spin clusters

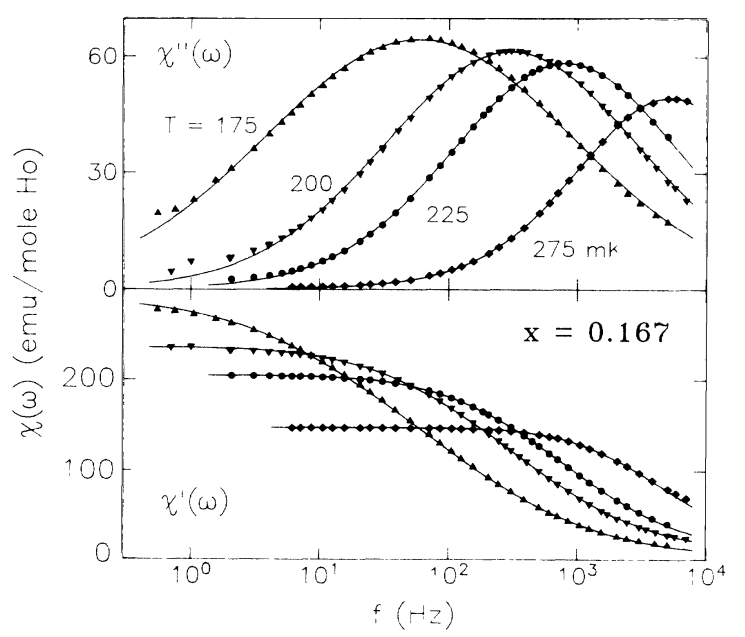

FIG. 8. Real $\left(\chi^{\prime}\right)$ and imaginary $\left(\chi^{\prime \prime}\right)$ parts of the magnetic susceptibility of $\mathrm{LiHo}_{0.167} \mathrm{Y}_{0.833} \mathrm{~F}_{4}$ vs frequency $f$ for several temperatures $T$. Solid lines are fits to a Gaussian distribution of energy barriers described in the text.

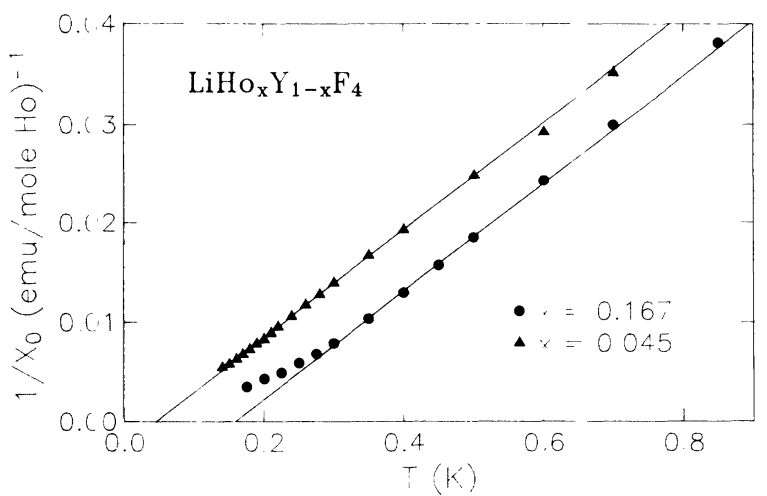

FIG. 9. The inverse dc susceptibility as a function of temperature. Solid lines are Curie-Weiss fits whose intercepts scale with dipole concentration $x$.

are present, ${ }^{36}$ a result directly confirmed by neutrondiffraction results presented below.

Dramatic and fundamental differences between the two glassy magnets emerge in the particulars of their spectroscopic response. How the magnetic dynamics change with $T$ is most easily found from a scaling plot of $\chi^{\prime \prime}(\omega)$, which by the fluctuation-dissipation theorem is simply $(\hbar \omega / k T) S(Q=0, \omega)$, where $S(Q, \omega)$ is the Fourier transform of the two-spin correlation function. Figure 10 shows such a plot, where the data have been scaled at each temperature by dividing the frequency by $f_{p}$ and the magnitude by $\chi^{\prime \prime}\left(f_{p}\right) . f_{p}$ is the frequency at which the peak in $\chi^{\prime \prime}(\omega)$ occurs. The symmetrical broadening with decreasing temperature for $x=0.167$ in the top half of the figure is in marked contrast to the behavior of the more dilute sample, $x=0.045$, shown in the bottom half of Fig. 10. The scaled susceptibility for this material is temperature independent at high scaled frequencies, and has a low-frequency tail whose width broadens with increasing $T$. The shapes of the curves on the scaling plots

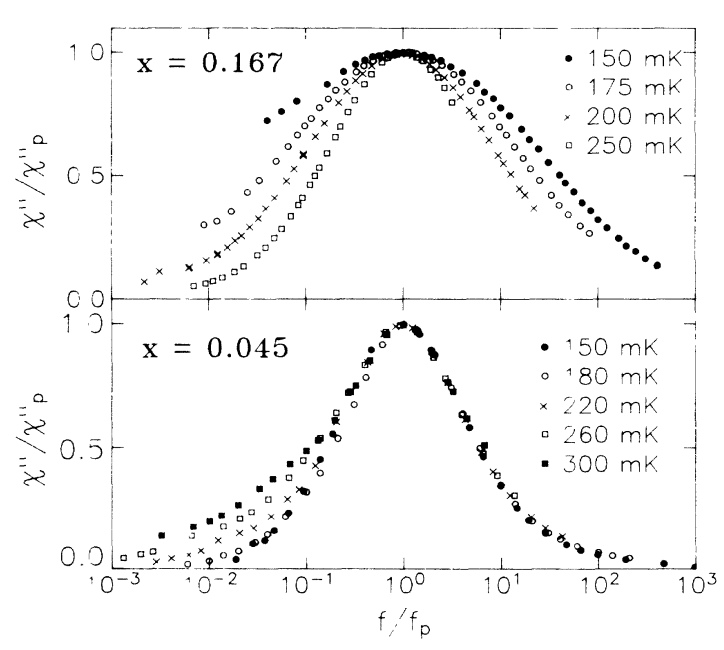

FIG. 10. Imaginary part of the magnetic susceptibility scaled by peak frequency and amplitude for $\mathrm{LiHo}_{x} \mathrm{Y}_{1-x} \mathrm{~F}_{4}$ with $x=0.167$ and $x=0.045$. $\chi^{\prime \prime}$ broadens (narrows) with decreasing temperature for the more concentrated (dilute) sample. 
are insensitive to the choice of demagnetization factor because of the scaling by $f_{p}{ }^{27}$

Increased (decreased) low-frequency weight with decreasing temperature reflects a broadening (narrowing) of the distribution of barriers to relaxation. Independent of any particular fitting form for $\chi(\omega, T)$, the scaling plot of $\chi^{\prime \prime}(\omega)$ provides a vivid illustration of the opposite manner in which the long-time relaxation processes evolve with $T$ for these two randomly diluted Ising glasses. It also implies a qualitatively different $T \rightarrow 0$ ground state simply as a function of dipole concentration.

In order to compare our data more directly to that in the spin-glass literature, we present a log-log plot of $\chi^{\prime \prime}(\omega)$ for the $x=0.045$ and 0.167 samples in Fig. 11 . $\chi^{\prime \prime}(\omega) \sim \omega^{\alpha}$ at low frequencies for both concentrations. While $\alpha$ decreases with decreasing $T$ for $x=0.167$, as is typically observed in spin-glass systems approaching $T_{g}$ from above, ${ }^{37} \alpha$ increases with decreasing $T$ for $x=0.045$.

We rely on a generalization of the Debye theory ${ }^{38}$ of dielectric relaxation to quantitatively analyze our data. The Debye model assumes that a single time $\tau$ governs all relaxation processes in the system. This leads to a complex susceptibility given by

$$
\chi(\omega)=\frac{\chi_{0}}{1-i \omega \tau}
$$

with a fixed width of 1.14 decades in frequency. A glassy system has multiple routes to relaxation, which is reflected in a much wider dynamic response. It is then appropriate to think in terms of a distribution of energy barriers to relaxation, $\rho\left(E_{B}\right)$, corresponding to the distribution of local environments for the spins, with each bar-

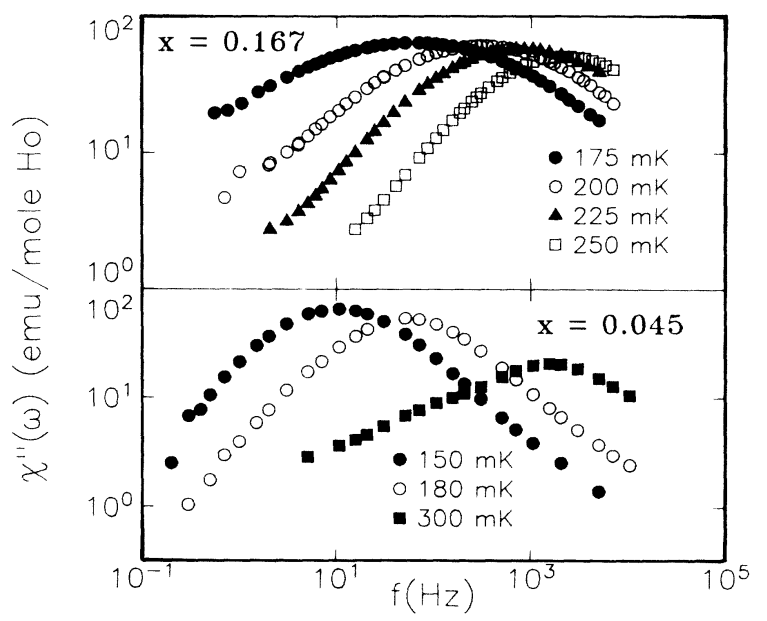

FIG. 11. Log-log plots of the imaginary part of the magnetic susceptibility vs frequency for $\mathrm{LiHo}_{x} \mathrm{Y}_{1-x} \mathrm{~F}_{4}$ for $x=0.167$ and $x=0.045$. The low-frequency susceptibility essentially obeys a power-law frequency dependence $\chi^{\prime \prime}(\omega) \sim \omega^{\alpha}$. The exponent $\alpha$ (the slope of $\chi^{\prime \prime}$ in this plot) increases with $T$ for $x=0.167$, but decreases as $T$ is increased for $x=0.045$. rier giving rise to a relaxation time via the Arrhenius form

$$
\tau\left(E_{B}\right)=\tau_{0} \exp \left(E_{B} / k T\right) .
$$

This gives for the susceptibility:

$$
\chi(\omega)=\chi_{0} \int_{0}^{\infty} \rho\left(E_{B}\right) \frac{d E_{B}}{1-i \omega \tau\left(E_{B}\right)} .
$$

The fits to the $x=0.167$ data shown in Fig. 8 come from a $\rho\left(E_{B}\right)$ given by

$$
\rho\left(E_{B}\right)=\frac{1}{\sqrt{2 \pi} \sigma} \exp \left[\frac{-1}{2}\left(\frac{E_{B}-E_{c}}{\sigma}\right)^{2}\right] .
$$

This Gaussian distribution of barriers has been used successfully in understanding other glassy systems, notably $(\mathrm{KBr})_{x}(\mathrm{KCN})_{1-x} \cdot{ }^{39}$ Since $\tau$ depends much more strongly on $E_{B}$ than on $\tau_{0}$, we assume that all its variation can be accounted for by variations in $E_{B}$, and fix $\tau_{0}=1 / f_{p}$. $\chi^{\prime \prime}$ was fit first by varying $\chi_{0}, \sigma$, and $E_{c} \cdot \chi^{\prime}$ was then fit with $\chi_{0}, \sigma$, and $E_{c}$ fixed at the values obtained from $\chi^{\prime \prime}$, varying only a small dc offset. We attribute this offset to a slight mismatch in the secondary coils. $E_{c}$ was temperature independent, giving a value of $E_{c}=2.05 \pm 0.03 \mathrm{~K}$ when averaged over the seven temperatures fit, in agreement with the value from the Arrhenius fit to the peak in $\chi^{\prime \prime}(\omega)$. Hence, the temperature dependence of $\chi(\omega)$ for $x=0.167$ can be represented in terms of only two parameters: $\chi_{0}$ and $\sigma$. Although generally successful in describing the data, we note that the Gaussian distribution cannot reproduce the asymptotic low-frequency power-law form of $\chi^{\prime \prime}(\omega)$.

Neither a Gaussian distribution of barriers nor any of the conventional asymmetric generalizations of Debye with which we are familiar can account for the behavior of the $x=0.045$ sample. We fit the susceptibility using ${ }^{40}$ instead a flat distribution of relaxation times with a large $\tau$ tail:

$$
\rho(\tau)= \begin{cases}\frac{(1-a)}{\tau_{c}} & \text { for } \tau<\tau_{c}, \\ \frac{a y \tau_{c}^{y}}{\tau^{y+1}} & \text { for } \tau>\tau_{c} .\end{cases}
$$

This gives rise to an asymmetric energy barrier distribution, dominated by exponential tails around a fixed barrier, $E_{c}$, that narrows as $T$ is reduced and describes well the data plotted in Fig. 7. The real and imaginary parts of $\chi(\omega)$ were fit separately; the two fits yielded the same values for $a, y, \chi_{0}$, and $\tau_{c}$ to within $10 \%$. We estimate error bars in each parameter for both the $x=0.045$ and $x=0.167$ functional forms by refitting the data with the parameter in question held fixed over a range around its best-fit value. This approach provides a measure of the curvature of the chi-squared surface as a function of each parameter. ${ }^{27}$ We define the error bars as the changes in the parameter which yield a $10 \%$ change in the meansquare deviation of the best fit from the data.

In order to directly compare the energy barrier distri- 
butions for the two magnetic glasses, described by different fitting forms, we plot their second moments, $\Delta E$, in Fig. 12. $\Delta E$ is defined by

$$
|\Delta E|^{2}=\int_{0}^{\infty} \rho\left(E_{B}\right)\left(E_{B}-E_{c}\right)^{2} d E_{B} .
$$

Note that for $x=0.167, \Delta E$ is equal to the fit parameter $\sigma$. The variation of $\Delta E$ with $T$ clearly shows the opposite trends in the two materials. The solid lines are guides to the eye. The scale and the exact temperature dependence of $\Delta E(T)$ may depend to some extent on the chosen fitting form or demagnetization approximation, but the direction in which $\Delta E$ evolves with $T$ is robust. The effect of sample geometry has been treated via a demagnetization correction, but there remains the possibility of shape altering the actual microscopic behavior. ${ }^{7,41} \mathrm{Fu}-$ ture investigations will address this issue.

$\mathrm{LiH}_{0.045} \mathrm{Y}_{0.955} \mathrm{~F}_{4}$ is unusual in the context of magnetic glasses, behaving as if it were heading for a zerotemperature ground-state dominated by a single barrier to relaxation. ${ }^{40}$ In contrast, $\mathrm{LiHo}_{0.167} \mathrm{Y}_{0.833} \mathrm{~F}_{4}$ behaves in a manner characteristic of spin glasses approaching their freezing temperature, $T_{g}$, from above, ${ }^{37}$ with a proliferation of barriers and concomitant broadening of $\chi^{\prime \prime}(\omega)$ as $T$ is reduced. If we assume critical slowing down at the spin-glass transition, then the average relaxation times are expected to diverge as ${ }^{42} \tau_{\mathrm{av}}=\tau_{0}\left[\left(T-T_{g}\right) / T_{g}\right]^{-z v}$, where we adopt Ogielski's definition, ${ }^{43}$

$$
\tau_{\mathrm{av}}=\lim _{\omega \rightarrow 0}(1 / \omega)\left[\chi^{\prime \prime}(\omega) / \chi^{\prime}(\omega)\right]
$$

$\tau_{0}$ is a microscopic relaxation time and $z v$ is the dynamic exponent. Fitting the data for the $x=0.167$ sample to this dynamical scaling form gives best-fit values $\tau_{0}=2.2 \pm 1.1 \times 10^{-3} \mathrm{~s}, T_{g}=0.10 \pm 0.01 \mathrm{~K}$ (open circle in Fig. 1) and $z v=7.4 \pm 0.6$. Monte Carlo simulations of Ising spin glasses ${ }^{43}$ find $z v=7.0 \pm 1.0$ when using $\tau_{\mathrm{av}}$. The agreement may be fortuitous, however, as our measurements extend out to $T / T_{g}=3$. We note that $\tau_{\mathrm{av}}$ is not well defined by the above form for the $x=0.045$ sample, preventing a dynamic scaling analysis in that case.

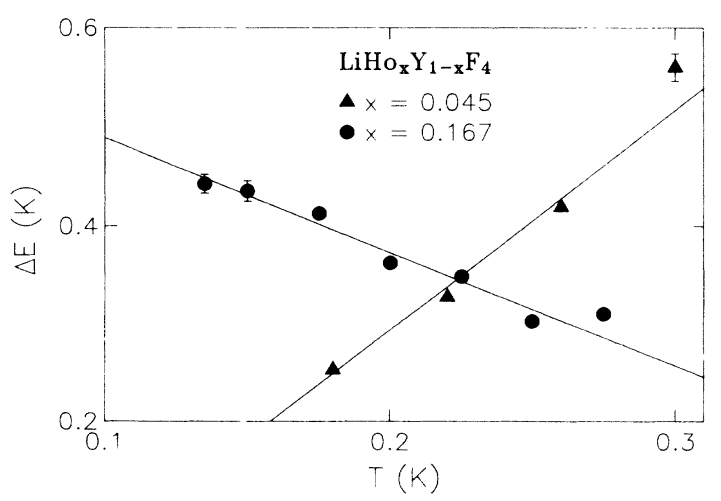

FIG. 12. The width of the distribution of energy barriers to relaxation, $\Delta E$, deduced from the ac susceptibility, evolves in an opposite manner with temperature, $T$, for the two glasses.

\section{Excitation spectrum}

The contrasting temperature evolution of the barriers to relaxation deduced from the two glasses' dynamic response implies markedly different ground states with dissimilar thermodynamic properties. We compare in Fig. 13 the specific heat, $C$, of the $x=0.045$ and $x=0.167$ samples normalized per mole of holmium for $0.13 \leq T \leq 1.5 \mathrm{~K}$. For both samples, $C(T)$ is dominated by a broad maximum, which is actually a background term due primarily to the moments $I_{i}\left(I=\frac{7}{2}\right)$ of the ${ }^{165} \mathrm{Ho}$ nuclei which are coupled to the electronic moments $\mathbf{J}_{i}$ via the hyperfine interaction $\sum A \mathbf{I}_{i} \cdot \mathbf{J}_{i}$. Mennenga et al. ${ }^{2}$ determined that for pure $\mathrm{LiHoF}_{4}, A / k=0.42 \mathrm{~K}$, which is comparable both to $x T_{C}(x=1)$ and the measuring temperatures for the present experiment. The solid line in Fig. 13(a) represents the hyperfine specific heat ${ }^{32}$ $C_{N}(T)$ for $\mathrm{Ho}^{3+}$ ions. The differences $\Delta C(T)=C(T)-C_{N}(T)$, shown in Fig. 13(b), clearly indicate interaction effects which are very different for the two samples. For $x=0.167, \Delta C(T)$ is typical of spin glasses with a broad maximum centered at $0.18 \mathrm{~K}$, a temperature of order the scaled Curie temperature $x T_{C}(x=1)=0.25 \mathrm{~K}$. Its amplitude is sufficiently large that for $0.13<T<1.0 \mathrm{~K}, \Delta C$ accounts for $85 \%$ of the entropy $(R \ln 2)$ associated with the (electronic) groundstate doublets via the thermodynamic identity,

$$
S\left(T_{2}\right)-S\left(T_{1}\right)=\int_{T_{1}}^{T_{2}}(\Delta C / T) d T .
$$

In contrast, in the same temperature range, an extraordinarily sharp Schottky-like (see below) peak dominates $\Delta C(T)$ for $x=0.045$. Its amplitude is well below that of the broad maximum for $x=0.167$, and indeed accounts for only $20 \%$ of the electronic entropy. ${ }^{44}$ As remarkable as the sharp peak at $0.3 \mathrm{~K}$ is the fact that $\Delta C$ is indistinguishable from zero at $T \approx 0.2 \mathrm{~K}$. Thus, to within experimental error, $C(T)$ for $x=0.045$ has no linear term, even

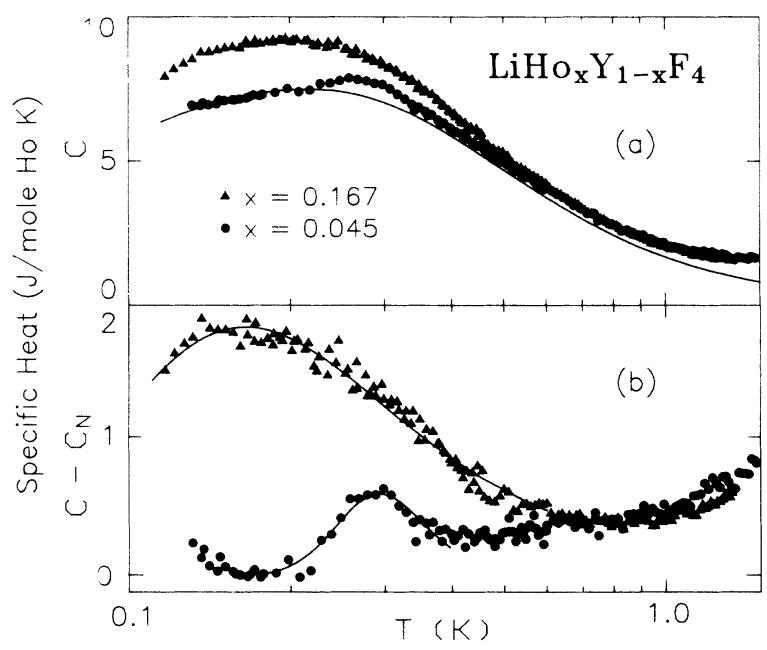

FIG. 13. (a) Total specific heat including the nuclear hyperfine contribution, $C_{N}$ (solid line, Ref. 32). (b) Electronic spin portion of the specific heat for the two magnetic glasses. Solid lines are fits to a Schottky-like form [Eq. (8)]. 
though such terms are present in most other structural and magnetic glasses. ${ }^{25,45,46}$ The data for the two samples essentially coincide at higher $T$, where $C$ is dominated by the Schottky contribution of excitations from the ground-state Ising doublet to the first-excited state singlet $9.4 \mathrm{~K}$ above. This high-temperature term should scale, as observed, with holmium concentration. The yttrium nuclear contribution only becomes consequential for temperatures below $0.04 \mathrm{~K}$ and the $T^{3}$ lattice contribution is only found ${ }^{2}$ to be significant for $T \geq 5 \mathrm{~K}$.

While $\chi^{\prime \prime}(\omega)$ can be analyzed to yield the spectrum of energy barriers, $C(T)$ is determined by the spectrum of excited states. The simplest spectrum consists of a discrete level with energy $E_{1}$ and degeneracy $n$ relative to that of the ground state. The corresponding specific heat is the generalized Schottky form,

$$
C / R=\frac{n E_{1}^{2}}{(k T)^{2}} \exp \left(\frac{-E_{1}}{k T}\right) /\left[1+n \exp \left(\frac{-E_{1}}{k T}\right)\right]^{2}
$$

Narrower peaks are associated with higher-excited state degeneracies. The solid lines in Fig. 13(b) correspond to the best fits of Eq. (8) to the data. The associated parameters are radically different for the two samples: $E_{1} / k=0.39 \mathrm{~K}$ and $n=0.8$ for $x=0.167(T \leq 0.6 \mathrm{~K})$, and $E_{1} / k=2.15 \mathrm{~K}$ and $n=890$ for $x=0.045$. Our results should not be literally interpreted as implying that the excited-state spectra consist of discrete levels. Instead, for $x=0.167$, we have simply shown that the spectrum of low-lying states is characterized by a typical energy of order $x k T_{C}(x=1)$ and a degeneracy similar to that of the ground state. In contrast, the sharp features in $C(T)$ for $x=0.045$ are due to excitations with energies of order $k T_{C}(x=1)$, i.e., the energy required to break a ferromagnetic bond between near neighbors. That such clusters are rare is apparent from the low entropy associated with the peaks. We note that an electronic contribution to the specific heat of the form $T^{-2}$ has been report$\mathrm{ed}^{46}$ in the insulating spin glass $\mathrm{Eu}_{x} \mathrm{Sr}_{1-x} S$ and identified as the tail of an (unseen) Schottky peak possibly arising from totally decoupled clusters created by frustration.

One way to measure $C(T)$ is to observe the response $\Delta T(t)$ of the sample temperature to a step in the heat applied to the sample. In general, the response is exponential, with a time constant $\tau \propto C(T)$. This is indeed the case at all measuring temperatures for $\mathrm{LiHo}_{x} \mathrm{Y}_{1-x} \mathrm{~F}_{4}$ with $x=0.167$. However, for $x=0.045$, there are increasingly serious deviations from the exponential law as $T$ is reduced below $100 \mathrm{mK}$. We show in Fig. 14 a semilog plot for both samples of $\Delta T$ versus time in units of the $1 / e$ decay time $\left(\sim 10^{3} \mathrm{sec}\right)$, normalized to $\Delta T$ at $t / \tau=0.1$. A straight line corresponds to the expected exponential decay. There is a rapid initial drop in $\Delta T$ at $t / \tau<<0.1$, which may be due to a time-dependent specific-heat characteristic of structural glasses ${ }^{47}$ or to thermal contact problems and is not understood at this time.

One way to parametrize a slower than exponential relaxation is via the so-called stretched exponential form,

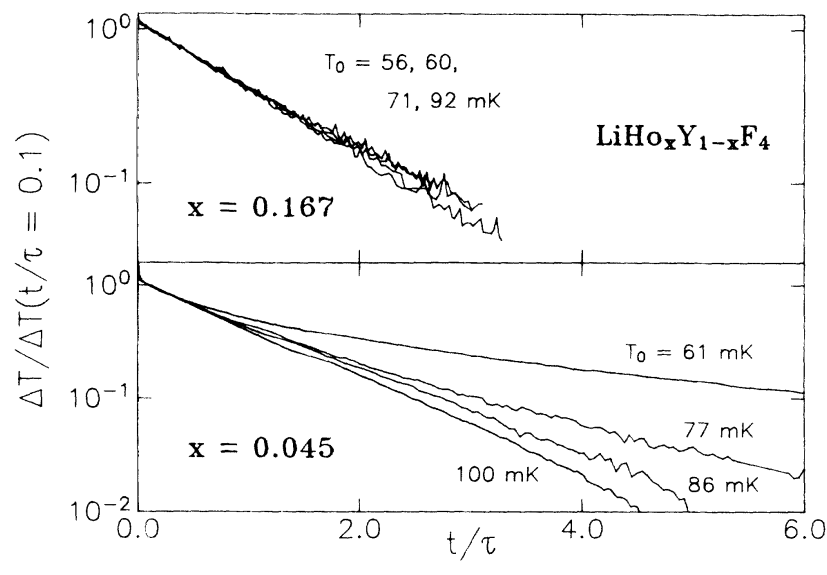

FIG. 14. Normalized change in temperature after a step in heat, $\Delta T$, vs time in units of the $1 / e$ decay time. For base temperature $T_{0}<100 \mathrm{mK}$, the thermal relaxation of $\mathrm{LiHo}_{0.045} \mathrm{Y}_{0.955} \mathrm{~F}_{4}$ becomes progressively slower than exponential. A purely exponential decay is found for $\mathrm{LiHo}_{0.167} \mathrm{Y}_{0.833} \mathrm{~F}_{4}$ for all $T_{0}$ measured.

$\Delta T \propto \exp (-t / \tau)^{\beta}$, with $0<\beta<1$. We fit the data for both $\mathrm{LiHo}_{0.045} \mathrm{Y}_{0.955} \mathrm{~F}_{4}$ and $\mathrm{LiHo}_{0.167} \mathrm{Y}_{0.833} \mathrm{~F}_{4}$ below $T=100 \mathrm{mK}$ to this form, and we plot the values of $\beta$ in Fig. 15. $\beta$ decreases smoothly from one with decreasing $T$ for $x=0.045$, but is consistent with one within the scatter for $x=0.167$. In the past, slower than exponential thermal relaxation has been associated with structural glasses, ${ }^{47}$ but not found in spin glasses. To the best of our knowledge, the current measurement represents the first observation of this effect for a disordered magnet. ${ }^{48}$ (Nonexponential decays of the magnetization are, of course, ubiquitous in such systems. ${ }^{26}$ ) Frequencydependent measurements ${ }^{49}$ of the specific heat of $(o-$ terphenyl $)_{1-x}(o \text {-phenylphenol })_{x}$ mixtures near the glass transition find a $\beta$ which extrapolates to zero at the glass transition temperature, $T_{g}$. If we assume that the

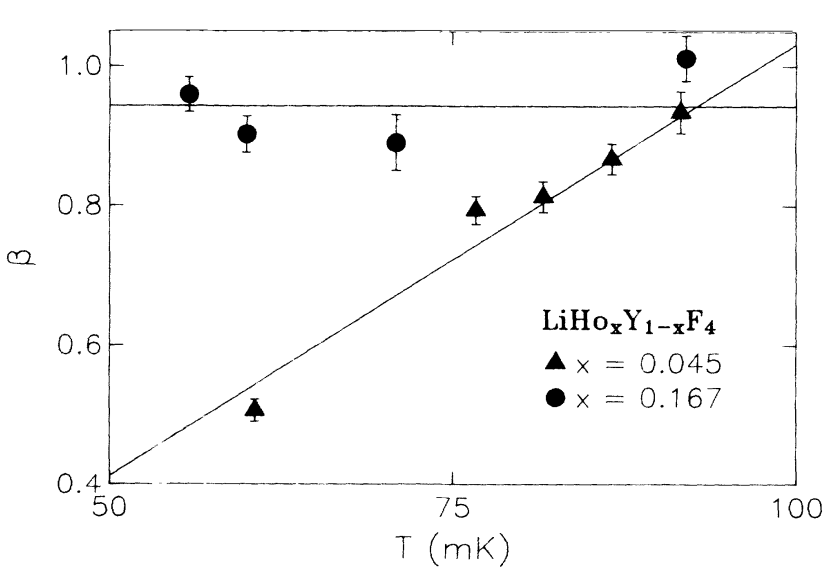

FIG. 15. Comparison of the thermal relaxation of the two dipole glasses for $T<100 \mathrm{mK}$, as parametrized by a stretched exponential form: $\Delta T \propto \exp (-t / \tau)^{\beta}$. Only the more dilute sample demonstrates a clear trend towards slower than exponential behavior. 
thermal relaxation parameter $\beta$ varies linearly with $T$, then $\beta$ extrapolates to zero at $T=0$ for $x=0.045$, implying that $T_{g} \sim 0$, consistent with our picture from the ac magnetic susceptibility.

\section{Magnetic correlations}

We conducted neutron scattering studies at Brookhaven in order to look directly at the spin correlations in the more concentrated glass. The neutron measurements cannot provide direct information about spin freezing ${ }^{50}$ because the energy scale for spin relaxation,

$$
\hbar \Gamma=\hbar / \tau_{\text {char }}=\left(4 \times 10^{-6} \mathrm{meV}\right) \exp [-2.2 / T(K)]
$$

from Eq. (2), is much smaller than the instrumental energy resolution $(\sim 0.3 \mathrm{meV})$. They do reveal, however, the typical size and shape of the slowly relaxing ferromagnetic clusters which contribute to the bulk $(q=0)$ ac response described above. Figures 16 and 17 show four scans through the magnetic diffuse scattering from $\mathrm{LiHo}_{0.167} \mathrm{Y}_{0.833} \mathrm{~F}_{4}$ at $T=0.15 \mathrm{~K}$. They follow the reciprocal space trajectories indicated by the dotted lines in the inset of Fig. 16. We express the momentum transfer

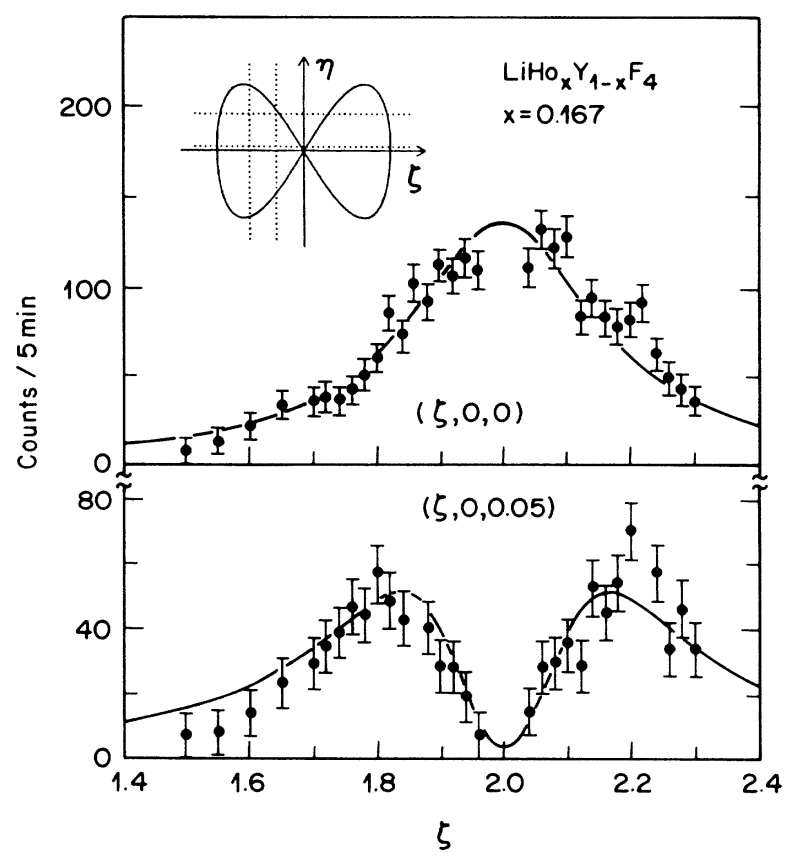

FIG. 16. Neutron-scattering intensity as a function of momentum transfer $\mathbf{Q}=(\zeta, 0, \eta)$ for $\eta=0$ (upper frame) and $0.05 \times c^{*}$ (lower frame). The off-axis scan shows the doublelobed profile characteristic of dipolar interactions. Solid lines are fits to the mean-field theory for a uniaxial dipolar-coupled magnet [Eqs. (9) and (10) in the text]. The small peak at $\zeta=2.22$ is the $(1,1,1)$ powder diffraction ring from the aluminum vacuum cans and radiation shield of the cryostat. Inset illustrates the reciprocal-space trajectories of the scans of this figure as well as those in Figs. 17 and 18.

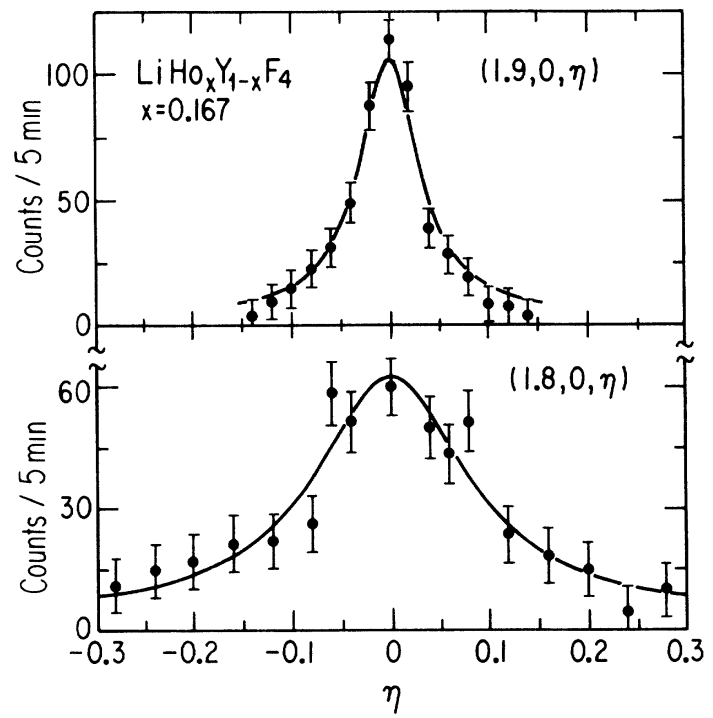

FIG. 17. Elastic scans along the Ising axis at $T=0.15 \mathrm{~K}$, showing that the scattering profile for $\mathbf{Q}=(\zeta, 0, \eta)$ widens with increasing distance of $\zeta$ from $2.0 \times a^{*}$. Solid lines are fits to the mean-field theory for a uniaxial dipolar-coupled magnet [Eqs. (9) and (10) in the text)].

in coordinates such that $\mathbf{Q}=(\zeta, 0, \eta)$, where $\zeta$ and $\eta$ are in units of $a^{*}=2 \pi / a=1.211 \AA^{-1}$ and $c^{*}=2 \pi / c$ $=0.579 \AA^{-1}$, respectively. Note that the dipoles are parallel to $(0,0,1)$. The $(\xi, 0,0)$ scan (upper frame of Fig. 16) passes directly through the $(2,0,0)$ nuclear Bragg point where magnetic Bragg scattering is observed for ferromagnetic samples of $\mathrm{LiHo}_{x} \mathrm{Y}_{1-x} \mathrm{~F}_{4}$. The most striking features of the data are (a) the occurrence of a minimum at $(2,0,0)$ for scans parallel to but slightly offset from the longitudinal $(\xi, 0,0)$ scan, and (b) the dramatic narrowing of the transverse scans $(\zeta, 0, \eta)$ as $\zeta$ approaches two. These features set dipolar-coupled systems apart from ordinary exchange-coupled magnets. The "bow-tie" shape of the constant intensity contours of the diffuse scattering (also indicated in the inset of Fig. 16) corresponds to FM clusters in real space which are elongated parallel to the (Ising) $c$ axis, along which the dipolar interaction is ferromagnetic, and pinched near their middles in the transverse directions, along which the dipolar interaction is antiferromagnetic.

In the quasielastic approximation, the magnetic cross section for unpolarized neutrons ${ }^{15}$ from Ising systems is

$$
\frac{d \sigma}{d \Omega}=\alpha\left[1-\left(\frac{Q_{z}}{Q}\right]^{2}\right] f^{2}(Q) \frac{\chi(\mathbf{Q}, T)}{\chi^{0}(T)},
$$

where $\alpha$ is the square of a coupling constant, $z$ refers to the Ising spin direction, $f(Q)$ is the form factor for $\mathrm{Ho}^{3+}, \chi(\mathbf{Q}, T)$ is the wave vector and temperaturedependent susceptibility, and $\chi^{0}(T)$ is the susceptibility of the system in the noninteracting limit. If we take $\mathbf{q}=\mathbf{Q}-\tau_{200}$, the deviation of $\mathbf{Q}$ from the $(2,0,0)$ reciprocal lattice vector, then, in the long-wavelength (small q) limit, the mean-field theory for a uniaxial, dipolar-coupled system ${ }^{17}$ gives 


$$
\chi^{-1}(\mathbf{Q}, T) \propto 1+\xi^{2}\left[q^{2}+g\left(\frac{q_{z}}{q}\right)^{2}\right] .
$$

$\xi$ is the correlation length in the plane perpendicular to the Ising axis, and $g$ is a measure of the anisotropy of the interaction. When allowed to float in the fits, $g$ showed no definite temperature dependence over the seven temperatures measured. Consequently, we refit the data using the average value $g=2.0$ (with uncertainty \pm 0.15 ), obtaining the curves shown in Figs. 16, 17, and 18.

The value $g=2.0$ is, as expected, somewhat larger than that observed for nondiluted systems. Mean-field calculations ${ }^{14}$ for $\mathrm{LiTbF}_{4}$ give $g=1.5$, while measurements of the critical scattering ${ }^{16}$ near $T_{C}$ find $g=1.3$. If one defines an average correlation length along the Ising axis, $\xi_{\|}$, as the inverse of the maximum width of the halfheight contour of the lobes of the scattering amplitude, then $\xi_{\|}=g^{1 / 2} \xi^{2}$. As the dipolar interaction between two spins in the plane is antiferromagnetic, in-plane ferromagnetic correlations must proceed indirectly via outof-plane neighbors. These will be affected more strongly by dilution than correlations along $c$, which are ferromagnetic without assistance from neighboring spins. This leads to a reduction of $\xi$ relative to $\xi_{\|}$as spins are removed from the systems, and, consequently, an increase in $g$.

Figure 18 shows that the $(\zeta, 0,0)$ scan becomes both more intense and narrower as $T$ is reduced, indicating that the FM correlations are growing even in the basal plane. We find from the fits to Eqs. (9) and (10) that $\xi^{2}$ increases from $2.6 \pm 1.7 \AA^{2}$ at $T=0.5 \mathrm{~K}$ to $20 \pm 4 \AA^{2}$ at $T=0.15(5) \mathrm{K}$. From the present neutron measurements it is unclear whether the growth in $\xi^{2}$ persists below $T \approx 0.2 \mathrm{~K}$. However, both the deviations from CurieWeiss behavior in the dc susceptibility (see Fig. 9) and the statistically insignificant increase in $\xi^{2}$ found between

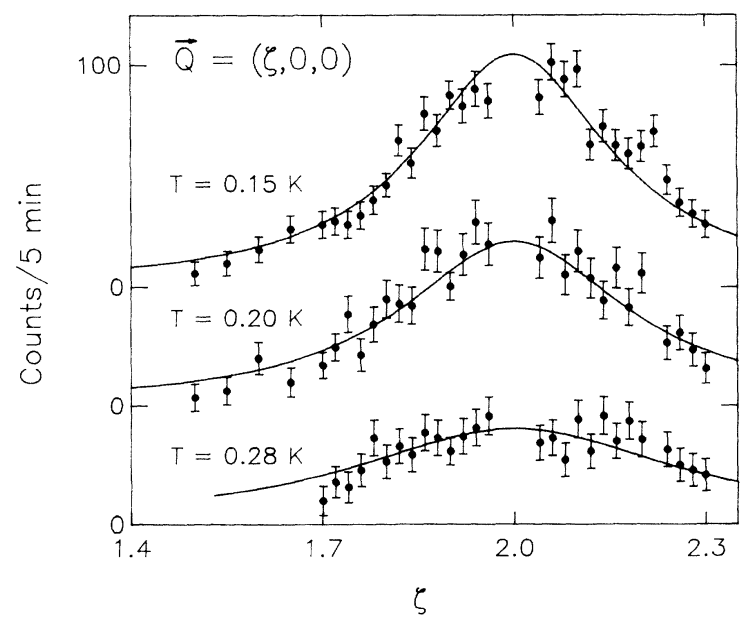

FIG. 18. Elastic scans as a function of $\zeta$ in $Q=(\xi, 0,0)$ for $\mathrm{LiHo}_{0.167} \mathrm{Y}_{0.833} \mathrm{~F}_{4}$ at a series of temperatures. Solid lines are fits to the mean-field theory for a unixial dipolar-coupled magnet [Eqs. (9) and (10) in the text]. The narrowing of the peaks with decreasing $T$ corresponds to an increasing in-plane ferromagnetic correlation length.
$T=0.2 \mathrm{~K}$ and $T=0.15 \mathrm{~K}$ argue against any rapid increase. The spin-glass state in $\mathrm{LiHo}_{0.167} \mathrm{Y}_{0.833} \mathrm{~F}_{4}$ then would involve collective freezing of sizable ferromagnetic clusters elongated along the Ising axis. The strong interactions between such clusters presumably give rise to the glassy behavior and the apparent freezing at this spin concentration. Sufficient interlocking inhibits the reorientation of the clusters into different spatial configurations which have essentially the same energy but opposite net moments. Furthermore, since the in-plane ferromagnetic correlations require assistance from out-ofplane neighbors, the formation of clusters should be concentration dependent. We believe that the $x=0.045 \mathrm{ma}$ terial is too dilute to have substantial in-plane ferromagnetic ordering (although the appropriate neutron experiments remain to be performed), and hence does not have the same type of cluster growth leading to freezing as we see for $x=0.167$. The substantially lower Curie-Weiss temperature for $x=0.045$ means that the fundamental FM correlated entities responsible for collective behavior must also be much smaller.

\section{DISCUSSION}

Pure $\mathrm{LiHoF}_{4}$ is a ferromagnet, with an essentially perfect (due to the long range of the dipolar interaction) mean-field transition ${ }^{14}$ at $T_{c}=1.53 \mathrm{~K}$. Random dilution of the magnetic $\mathrm{Ho}^{3+}$ sites with nonmagnetic $\mathrm{Y}^{3+}$ for spin concentrations down to at least $x=0.46$ preserves the ferromagnetism, with a critical temperature given by a simple scaling with $x$ of the $T_{c}$ of the pure system. This disordered ferromagnet awaits closer investigation to test the predictions ${ }^{20}$ of novel critical exponents for the random, dipolar-coupled Ising magnet at the marginal dimensionality. Neutron-scattering studies $^{51}$ of $\mathrm{LiHo}_{0.3} \mathrm{Y}_{0.7} \mathrm{~F}_{4}$ find a ferromagnetic ground state, but with a $T_{c} 20 \%$ below the mean-field prediction and with a saturation magnetic moment per rare-earth ion only $60 \%$ of that observed in the undiluted material. For $x \leq 0.167$, magnetic glassiness ensues, but with what appear to be fundamentally different regimes of glassy response as a function of dipole concentration. The behavior of $\mathrm{LiHo}_{x} \mathrm{Y}_{1-x} \mathrm{~F}_{4}$ with $x=0.167$ is consistent with our expectations for a canonical spin glass, ${ }^{52}$ as evidenced by the temperature evolution of the low-frequency magnetic susceptibility, equilibrium specific heat, and thermal relaxation. For $x=0.045$, however, the more dilute set of interacting dipoles behaves as if reducing the temperature reduces their interactions. This behavior is completely different from that for spin glasses, which, according to theory, ${ }^{21}$ should also include arbitrarily dilute dipolarcoupled magnets.

While we do not have a detailed, microscopic explanation for the behavior of the more dilute sample, $x=0.045$, the following simple model reproduces the effects we observe. The ac susceptibility data indicate that as the system approaches its zero-temperature ground state, spin relaxation is dominated by a single barrier, $E_{c}$. This is consistent with a picture in which the ground state for $x=0.045$ consists of spin clusters with zero net dipole moment. ${ }^{40}$ The molecular field in which 
spin reorientation occurs is therefore effectively zero. As $T$ is increased, some clusters are excited into states with nonzero moments, which then act on spins in other clusters. Thus, the distribution of molecular fields, and hence the distribution of energy barriers to spin relaxation, will broaden with increasing temperature.

The reorientation of the isolated clusters involves flips of individual dipoles, an energy of order the nearestneighbor dipole-dipole interaction energy, $E_{B} / k \sim 2 \mathrm{~K}$ from the Arrhenius law. Indeed, we find from our analysis of the specific heat of the $x=0.045$ sample an energy $E_{1} / k=2.15 \mathrm{~K}$, the energy of a spin flip in a nearest-neighbor pair. This splitting between ground and excited states is much greater than the measuring temperature. In contrast, the specific heat of the $x=0.167$ sample is characterized by a lower energy, $E_{1} / k=0.39 \mathrm{~K}$, presumably due to collective motions of many spins within an extended cluster where frustrated regions can reorient into spatially distinct configurations of roughly equivalent energies. Here, the splitting between "ground" and "excited" states is of order $T$ and it is difficult to make a distinction between both highly degenerate levels. The characteristic energy $E_{1}$ must also be of order the molecular field associated with the relatively large ferromagnetic clusters found by our neutron measurements.

We attempt to illustrate the distinctions between the different ground states in Fig. 19. The upper panel represents a characteristic (Ising) spin configuration, the middle panel the distribution, $D_{0}(E)$, of single-spin-flip energies $E$ for the ground state, and the lower panel the distribution, $D_{1}(E)$, of energies for spin flips given that one spin has reoriented. Assuming that individual spin flips are good representations of the excited eigenstates of the system, the specific heat is dominated at low $T$ by

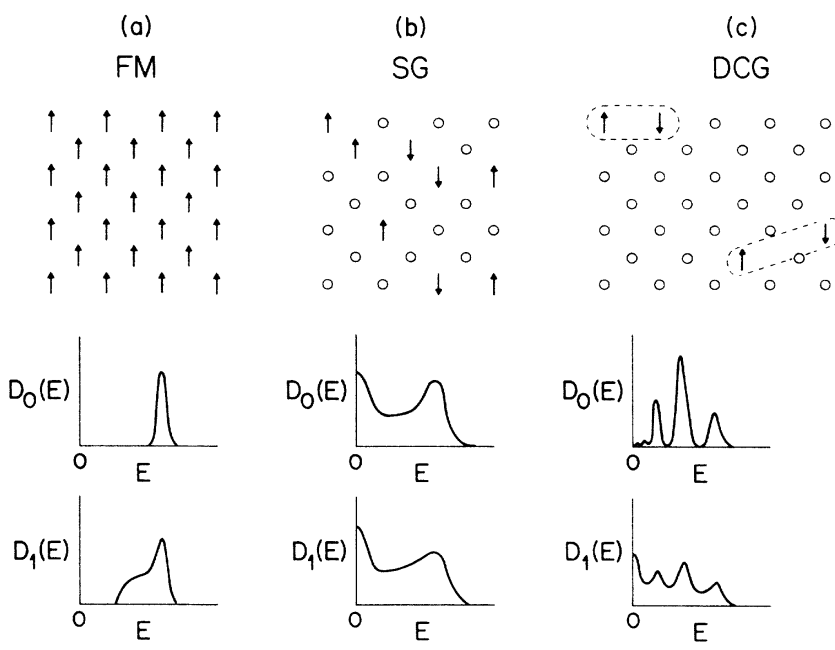

FIG. 19. Schematic of representative spin configurations, and the densities of states $D_{0}(E)$ and $D_{1}(E)$, for single spin flips relative to the unperturbed ground state and relative to the state with one spin already flipped, respectively, as a function of decreasing concentration of dipoles. Open circles in upper frames represent nonmagnetic ions. FM denotes ferromagnet, SG denotes spin glass, and DCG denotes decoupled cluster glass.
$D_{0}(E)$. As $T$ approaches zero, magnetic relaxation is also related to $D_{0}(E)$. In the case of the ferromagnet, all spins are aligned with a narrow $D_{0}(E)$ centered about $E \sim k T_{C}(x=1)$. After a spin flip, there are lower-energy excitations present in the vicinity of that spin which leads to the low-energy broadening in $D_{1}(E)$. In the case of the spin glass, spins point randomly with zero net magnetization. One thus expects a broad ground-state density of states, $D_{0}(E)$, with a characteristic energy width of order $x k T_{C}(x=1)$. Local changes in spin orientations should not appreciably affect the density of states; hence the similarity between $D_{1}(E)$ and $D_{0}(E)$. Finally, for the case of the "decoupled cluster glass" (DCG), we have explained our data with essentially isolated clusters with zero net moments, whose coupling increases with $T$ as a nearest neighbor is excited into a ferromagnetic configuration. Here, there are narrow peaks in $D_{0}(E)$ at energies from zero to $k T_{C}(x=1)$. The coupling between clusters after a spin flip makes $D_{1}(E)$ for the DCG resemble $D_{1}(E)$ for the spin-glass state. This coupling, unique to systems with long-range interactions, is responsible for the broadening of the distribution of barriers to relaxation as $T$ is increased. For the classic case of decoupled clusters in a system with short-range interactions, namely a near-neighbor exchange-coupled magnet below its percolation threshold, $D_{0}(E)$ and $D_{1}(E)$ are qualitatively the same: Both consist of sums of finite numbers of delta functions.

The DCG state is most unusual in view of the longrange nature of the dipolar interaction. Whether systems of interacting spins can display ground states which are not frozen in the collective sense has been a fundamental question in the study of magnetism. When quantum mechanics is considered such systems can be found, as in the case of the one-dimensional antiferromagnet. For disordered $s=\frac{1}{2}$ Heisenberg antiferromagnets, local singlet formation also preempts spin freezing. ${ }^{53}$ However, for classical spins, finite temperature freezing is generally expected if the range of the interactions exceeds a suitably defined spacing between spins.

Data on other relatively dense classical spin systems also point to the possibility of freezing only at zero temperature. For example, searches for a spin-glass phase due to dipolar interactions between the $s=\frac{7}{2}$ moments on $\mathrm{Eu}^{2+}$ in dilute $\mathrm{Eu}_{x} \mathrm{Sr}_{1-x} \mathrm{~S}$ were unsuccessful. ${ }^{54}$ Similarly, a variety of Ising rubies $\left[\left(\mathrm{Cr}_{x} \mathrm{Al}_{1-x}\right) \mathrm{O}_{3}\right]$ have been shown $^{55}$ to possess at best a $T_{g}=0$ spin-glass transition. In the case of $\mathrm{LiHo}_{x} \mathrm{Y}_{1-x} \mathrm{~F}_{4}$, we have shown that it is possible to study the evolution from DCG to SG to FM simply as a function of dipole concentration.

Finally, with a view towards relating the magnetic (and thermal) relaxation data to the specific-heat results, it is of interest to consider the mean degeneracy $N$ of occupied states. For $T=0, N$ is simply the degeneracy $N_{0}$ of the ground state, while for finite $T, N$ can deviate from $N_{0}$ as given by the thermal occupation of excited states. In the case of the single level assumed in the derivation of Eq. (8),

$$
N / N_{0}=\left\langle n_{\text {occ }}\right\rangle=\frac{1+n^{2} \exp \left(-E_{1} / k T\right)}{1+n \exp \left(-E_{1} / k T\right)} .
$$


If $n<1, N$ decreases with increasing $T$, while if $n>1, N$ increases with $T$. Thus, if our parametrization of $C(T)$ were literally correct, we would conclude that $\partial N / \partial T<0$ and $>0$ for the $x=0.167$ and 0.045 samples, respectively. When we consider that magnetic relaxation is slower for large degeneracies, it follows that the scaled lowfrequency susceptibilities $\chi^{\prime \prime}\left(f / f_{p}\right)$ should be enhanced with decreasing $T$ for $x=0.167$, and increasing $T$ for $x=0.045$. Hence, we have at least a qualitative understanding of the opposing trends in the ac susceptibility. Analogous arguments can be made for the thermal relaxation. Specifically, a particular temperature is characterized by a particular occupation of excited states. If the excited states do not stand in one-to-one correspondence with the states whose energies are close (within kT) to the ground-state energy, achieving the correct occupation (after application of heat) for a new equilibrium will typically be a multistep process involving several rates. For example, when the excited states are clustered in relatively narrow manifolds, as they are for $x=0.045$, the first of these rates involves the transition between the wellseparated ground and excited state manifolds, and the others are due to the "settling" process within the excited-state manifold. In agreement with experiment, this physical picture implies that for the spin-glass sample, where the relative degeneracy $n \sim 1$, the thermal relaxation should be exponential, while for $x=0.045$, the relative excited-state degeneracies $n \gg 1$, and the thermal relaxation would more likely be nonexponential.

\section{CONCLUSIONS}

We have studied the randomly diluted dipolar-coupled system $\mathrm{LiHo}_{x} \mathrm{Y}_{1-x} \mathrm{~F}_{4}$ and find dramatically different properties for $x=0.045$ and 0.167 . For the larger concentration, behavior typical of a spin glass is observed, while for $x=0.045$, we have discovered nonexponential thermal relaxation, a sharp Schottky-like peak in the specific heat, and a scaled low-frequency magnetic response which becomes smaller with decreasing $T$. We have given heuristic arguments as to the self-consistent nature of these properties. Their fundamental physical origin remains to be precisely specified. Nevertheless, a ground state consisting of decoupled, finite spin clusters which become coupled when in their (thermally) excited states, would yield the newly discovered phenomena for $x=0.045$. As $x$ is increased, the excited state manifolds would intermingle with the ground-state manifold, and yield a conventional spin glass whose response is dominated by a single infinite cluster. Neutron-scattering studies of the $x=0.167$ material show the growth of ferromagnetic clusters, and suggest that freezing is governed by the growth of these clusters in the plane perpendicular to the Ising axis. It is our hope that the study of this microscopically clean model system will open new avenues to the understanding of the complex macroscopic behavior of glasses.

\section{ACKNOWLEDGMENTS}

The work at The University of Chicago was supported by the National Science Foundation (NSF) Materials Research Laboratory under Grant No. 88-19860. We are grateful to S. R. Nagel, D. L. Stein, and C. Yu for helpful discussions. We thank S. Shapiro and J. Axe for their generous hospitality at Brookhaven National Laboratory (BNL). Neutron scattering at BNL was supported by the Division of Materials Science, U.S. Department of Energy, under Contract No. DE-AC02-76CH0016. D. H. Reich submits this paper in partial fulfillment of the requirements for the Ph.D. degree at The University of Chicago. Thanks are also due to the Aspen Center for Physics, where part of the work leading to this paper was done.
"Present address: AT\&T Bell Laboratories, Murray Hill, New Jersey 07974.

†Present address: Physics Department, University of California, Santa Cruz, California 95064.

${ }^{1}$ C. Keller and H. Schmutz, J. Inorg. Nucl. Chem. 27, 900 (1965).

${ }^{2}$ G. Mennenga, L. J. de Jongh, and W. J. Huiskamp, J. Magn. Magn. Mater. 44, 59 (1984).

${ }^{3}$ P. E. Hansen, T. Johansson, and R. Nevald, Phys. Rev. B 12, 5315 (1975).

${ }^{4}$ D. H. Reich, T. F. Rosenbaum, G. Aeppli, and H. Guggenheim, Phys. Rev. B 34, 4956 (1986).

${ }^{5}$ L. Onsager, J. Am. Chem. Soc. 58, 1486 (1936).

6J. H. Van Vleck, J. Chem. Phys. 5, 320 (1937).

${ }^{7}$ J. M. Luttinger and L. Tisza, Phys. Rev. 70, 954 (1946).

${ }^{8}$ M. H. Cohen and F. Keffer, Phys. Rev. 99, 1135 (1955).

${ }^{9}$ A. Aharony, Phys. Rev. B 8, 3363 (1973).

${ }^{10}$ A. Aharony and M. E. Fisher, Phys. Rev. B 8, 3323 (1973).

${ }^{11}$ L. M. Holmes, T. Johansson, and H. J. Guggenheim, Solid State Commun. 12, 993 (1973).

${ }^{12}$ J. Als-Nielsen, L. M. Holmes, and H. J. Guggenheim, Phys. Rev. Lett. 32, 610 (1974).
${ }^{13}$ L. M. Holmes, F. Hulliger, H. J. Guggenheim, and J. P. Maita, Phys. Lett. 50A, 163 (1974).

${ }^{14}$ G. Ahlers, A. Kornblit, and H. J. Guggenheim, Phys. Rev. Lett. 34, 1227 (1975).

${ }^{15}$ L. M. Holmes, J. Als-Nielson, and H. J. Guggenheim, Phys. Rev. B 12, 180 (1975).

16J. Als-Nielsen, L. M. Holmes, F. Krebs Larsen, and H. J. Guggenheim, Phys. Rev. B 12, 191 (1975).

${ }^{17}$ J. Als-Nielsen, Phys. Rev. Lett. 37, 1161 (1976).

18J. A. Griffin, M. Huster, and R. J. Folweiler, Phys. Rev. B 22, 4370 (1980).

${ }^{19}$ A. Aharony and B. I. Halperin, Phys. Rev. Lett. 35, 1308 (1975).

${ }^{20}$ A. Aharony, Phys. Rev. B 13, 2092 (1976).

${ }^{21}$ A. Aharony and M. J. Stephen, J. Phys. C 14, 1665 (1981).

${ }^{22}$ P. Beauvillain, J. Seiden, and L. Laursen, Phys. Rev. Lett. 45, 1362 (1980).

${ }^{23}$ J. A. Griffin, J. J. Folkins, and D. Gabbe, Phys. Rev. Lett. 45, 1887 (1980).

${ }^{24}$ R. W. Youngblood, G. Aeppli, J. D. Axe, and J. A. Griffin, Phys. Rev. Lett. 49, 1724 (1982).

${ }^{25}$ See, for example, H. Maletta and P. Convert, Phys. Rev. Lett. 
42, 108 (1979).

${ }^{26}$ See K. Binder and A. P. Young, Rev. Mod. Phys. 58, 801 (1986) for an extensive review.

${ }^{27}$ D. H. Reich, Ph.D. thesis, The University of Chicago, 1988 (unpublished).

${ }^{28}$ J. F. Schooley, G. A. Evans, Jr., and R. J. Soulen, Jr., Cryogenics 20, 193 (1980); R. J. Soulen, Jr., J. F. Schooley, and G. A. Evans, Jr., Rev. Sci. Instrum. 44, 1537 (1973).

${ }^{29}$ N. O. Birge and S. R. Nagel, Rev. Sci. Instrum. 58, 1164 (1987); P. K. Dixon, ibid. 60, 3329 (1989).

${ }^{30}$ K. B. Lyons, P. A. Fleury, T. J. Negran, and H. L. Carter, Phys. Rev. B 36, 2465 (1987).

${ }^{31}$ We approximate the shape of the sample by prolate ellipsoids of revolution. The ratio of the major to the minor axes is 1.4 , 1.7 , and 1.8 for the $x=0.46,0.167$, and 0.045 samples, respectively. By this approximation, we find $\chi$ of the $x=0.46$ sample at $93 \%$ of its demagnetizing limit, $\chi$ of the $x=0.167$ sample at $67 \%$ of its demagnetizing limit, and $\chi$ of the $x=0.045$ sample at $21 \%$ of its demagnetizing limit. See A. M. Morrish, The Physical Principles of Magnetism (Wiley, New York, 1965), Appendix II, and R. M. Bozorth and D. M. Chapin, J. Appl. Phys. 13, 320 (1942).

${ }^{32}$ V. A. Atsarkin, Phys. Lett. A 130, 492 (1988).

${ }^{33}$ See also L. Lundgren, P. Svedlindh, P. Nordblad, and O. Beckman, Phys. Rev. Lett. 51, 911 (1983).

${ }^{34}$ An Arrhenius law for the peak frequency does not necessarily indicate an absence of freezing at finite temperature. See, for example, the work on cobalt aluminoscilicate spin glasses: $L$. E. Wenger, in Proceedings of the Heidelberg Colloquium on Spin Glasses, Vol. 192 of Lecture Notes in Physics, edited by J. L. van Hemmen and I. Morgenstern (Springer, Berlin, 1983), p. 60 .

${ }^{35}$ R. B. Griffiths, Phys. Rev. Lett. 23, 17 (1969).

${ }^{36}$ K. V. Rao, M. Fahnle, E. Figueroa, O. Beckman, and L. Hedman, Phys. Rev. B 27, 3104 (1983); A. F. J. Morgownik and J. A. Mydosh, Solid State Commun. 47, 321 (1983).

${ }^{37}$ D. Huser, L. E. Wenger, A. J. van Duyneveldt, and J. A. Mydosh, Phys. Rev. B 27, 3100 (1983); C. C. Paulsen, S. J. Williamson, and H. Maletta, Phys. Rev. Lett. 59, 128 (1987).

${ }^{38}$ P. Debye, Polar Molecules (Chemical Catalogue, New York, 1929), p. 94.
${ }^{39}$ N. O. Birge, Y. H. Jeong, S. R. Nagel, S. Bhattacharya, and S. Susman, Phys. Rev. B 30, 2306 (1984).

${ }^{40}$ D. H. Reich, T. F. Rosenbaum, and G. Aeppli, Phys. Rev. Lett. 59, 1969 (1987).

${ }^{41}$ S. J. Knak Jensen and K. Kjaer, J. Phys. Condens. Matter 1, 2361 (1989).

${ }^{42}$ P. C. Hohenberg and B. I. Halperin, Rev. Mod. Phys. 49, 435 (1977).

${ }^{43}$ A. T. Ogielski, Phys. Rev. B 32, 7834 (1985).

${ }^{44}$ Calorimetry for $0.1<T<0.13 \mathrm{~K}$ using the relaxational method alone indicates a possible second, smaller and even narrower peak, which would account for another $5 \%$ of the electronic entropy.

${ }^{45}$ R. O. Pohl, in Amorphous Solids: Low Temperature Properties, edited by W. A. Phillips (Springer-Verlag, Berlin, 1981), p. 27.

${ }^{46}$ D. Meschede, F. Steglich, W. Felsch, H. Maletta, and W. Zinn, Phys. Rev. Lett. 44, 102 (1980).

${ }^{47}$ M. T. Loponen, R. C. Dynes, V. Narayanamurti, and J. P. Garno, Phys. Rev. Lett. 45, 457 (1980); Phys. Rev. B 25, 1161 (1982); J. Zimmerman and G. Weber, Phys. Rev. Lett. 46, 661 (1981); M. Meissner and K. Spitzmann, ibid. 46, 265 (1981).

${ }^{48}$ Nonexponential decay of energy flow has been reported when a remanent magnetization is present: A. Berton, J. Chaussy, J. Odin, H. Peyrard, J. J. Prejean, and J. Souletie, Solid State Commun. 37, 241 (1981).

${ }^{49}$ P. K. Dixon and S. R. Nagel, Phys. Rev. Lett. 61, 341 (1988); J. Chem. Phys. 90, 3885 (1989).

${ }^{50} \mathrm{G}$. Aeppli, J. J. Hauser, G. Shirane, and Y. J. Uemura, Phys. Rev. Lett. 54, 843 (1985).

${ }^{51}$ K. Kjaer, J. Als-Nielsen, I. Laursen, and F. Krebs Larsen, J. Phys. Condens. Matter 1, 5743 (1989).

${ }^{52}$ The low attempt frequency makes it difficult to pursue studies of the divergence of the nonlinear susceptibility, which would be a more definitive indication of a true spin-glass transition. See, for example, L. P. Levy and A. T. Ogielski, Phys. Rev. Lett. 57, 3288 (1986).

${ }^{53}$ R. N. Bhatt and P. A. Lee, Phys. Rev. Lett. 48, 344 (1982).

${ }^{54}$ J. Kötzler and G. Eiselt, Phys. Rev. B 25, 3207 (1982).

${ }^{55}$ J. Kötzler, J. Hesse, H. P. Tödter, and G. Eiselt, Z. Phys. B 68, 451 (1987). 